Article

\title{
Trace Metal and Cd Isotope Systematics of the Basal Datangpo Formation, Yangtze Platform (South China) Indicate Restrained (Bio)Geochemical Metal Cycling in Cryogenian Seawater
}

\author{
Simon V. Hohl ${ }^{1,2, *}{ }^{\oplus}$, Shao-Yong Jiang ${ }^{3, *}$, Sebastian Viehmann ${ }^{4} \oplus$, Wei Wei ${ }^{5}{ }^{\oplus}$, Qian Liu ${ }^{2}$, \\ Hai-Zhen Wei ${ }^{2}$ and Stephen J.G. Galer ${ }^{6}$ \\ 1 State Key Laboratory of Marine Geology, School of Ocean and Earth Science, Tongji University, Shanghai \\ 200092, China \\ 2 State Key Laboratory for Mineral Deposits Research, Department of Earth Sciences, Nanjing University, \\ Nanjing 210093, China \\ 3 State Key Laboratory of Geological Processes and Mineral Resources, School of Earth Resources, China \\ University of Geosciences, Wuhan 430074, China \\ 4 Department of Geodynamics and Sedimentology, University of Vienna, Wien 1090, Austria \\ 5 School of Earth and Space Sciences, University of Science and Technology, Hefei 471023, China \\ 6 Climate Geochemistry Department, Max Planck Institute for Chemistry, Mainz 55128, Germany \\ * Correspondence: sv_hohl@tongji.edu.cn (S.V.H.); shyjiang@cug.edu.cn (S.-Y.J.)
}

Received: 9 December 2019; Accepted: 6 January 2020; Published: 19 January 2020

\begin{abstract}
The behaviour of bioavailable trace metals and their stable isotopes in the modern oceans is controlled by uptake into phototrophic organisms and adsorption on and incorporation into marine authigenic minerals. Among other bioessential metals, $\mathrm{Cd}$ and its stable isotopes have recently been used in carbonate lithologies as novel tracer for changes in the paleo primary productivity and (bio)geochemical cycling. However, many marine sediments that were deposited during geologically highly relevant episodes and which, thus, urgently require study for a better understanding of the paleo environment are rather composed of a mixture of organic matter (OM), and detrital and authigenic minerals. In this study, we present $\mathrm{Cd}$ concentrations and their isotopic compositions as well as trace metal concentrations from sequential leachates of OM-rich shales of the Cryogenian basal Datangpo Formation, Yangtze Platform (South China). Our study shows variable distribution of conservative and bioavailable trace metals as well as $\mathrm{Cd}$ isotope compositions between sequential leachates of carbonate, $\mathrm{OM}$, sulphide, and silicate phases. We show that the $\mathrm{Cd}$ isotope compositions obtained from OM leachates can be used to calculate the ambient Cryogenian surface seawater of the restricted Nanhua Basin by applying mass balance calculations. By contrast, early diagenetic Mn carbonates and sulphides incorporated the residual $\mathrm{Cd}$ from dissolved organic matter that was in isotopic equilibrium with deep/pore waters of the Nanhua Basin. Our model suggests that the $\mathrm{Cd}$ isotopic composition of surface seawater at that time reached values of modern oxygenated surface oceans. However, the deep water $\mathrm{Cd}$ isotope composition was substantially heavier than that of modern fully oxygenated oceans and rather resembles deep waters with abundant sulphide precipitation typical for modern oxygen minimum zones. This argues for incomplete recycling of $\mathrm{Cd}$ and other bioavailable metals shortly after the Sturtian glaciation in the redox stratified Cryogenian Nanhua Basin. Our study highlights the importance of sequential leaching procedures when dealing with impure authigenic sediments such as OM-rich carbonates, mudstones, or shales to achieve reliable trace metal concentrations and $\mathrm{Cd}$ isotope compositions as proxies for (bio)geochemical metal cycling in past aquatic systems.
\end{abstract}

Keywords: Cryogenian; Cd isotopes; (bio)geochemical metal cycling; paleo productivity; sequential leaching; Datangpo Formation; Yangtze Platform 


\section{Introduction}

\subsection{The Cryogenian Datangpo Formation and the Emergence of Substantial Algae Growth}

The Neoproterozoic is one of the geologically most fascinating eras in Earth's history as it witnessed profound paleoenvironmental changes such as the breakup of the Rodinia supercontinent [1], various alternations between icehouse and greenhouse conditions [2,3], deep ocean oxygenation $[4,5]$, and the first appearance of macroscopic multicellular organisms [6-9]. Within the Neoproterozoic Era, the Cryogenian Period is of particular interest for (geo)biologists as sedimentary deposits during nonglacial intervals already showed the first geochemical evidence for a redox-stratified ocean with an oxidized surface layer $[10,11]$. This paleoenvironment is widely discussed as the habitat set up for the emergence of the first animals in the following Ediacaran Period [12-14]. Furthermore, biomarkers from Cryogenian strata indicate that demosponges [15] and algae [16] became important in Earth's oceans by that time. These two organisms presumably led to subsequent oxygenation of the deep oceans by filtration and recycling of sinking organic matter $(\mathrm{OM})$ as well as by photosynthesis, and, in the case of algae, as efficient nutrient recyclers and main food source for the emerging animal classes in the late Neoproterozoic following the Marinoan glaciation [17]. It was possibly the combination of extreme environmental conditions during the Cryogenian (low $\mathrm{pO}_{2}$ and periodic widespread ice coverage of the ocean, blocking the light/energy source for phototrophic organisms) that triggered evolutionary inventions which took place in the following Ediacaran and Cambrian periods. However, the research community still lacks a general understanding of if nutrient conditions in the Cryogenian ocean were generally unfavourable for life and which role primary producers played during interglacial periods as these processes may have led the foundation for the following 'explosion' of life.

The marine sediments of the Datangpo Formation (Fm.) were deposited during the nonglacial interval between the presumably globally occurring, low-latitude Sturtian and Marinoan glaciations in the intra-Yangtze Platform Nanhua Basin [18]. These two glaciations are also locally known as the Tiesi'ao and Nantuo glaciation, respectively. Magmatic zircons sampled in ash beds at the base and top of the interglacial Datangpo Fm. have been dated to $663 \pm 4 \mathrm{Ma}$ (isotope dilution thermal ionization mass spectrometry (ID TIMS) U-Pb age) and $654.5 \pm 3.8 \mathrm{Ma}$ (sensitive high-resolution ionprobe (SHRIMP) U-Pb age) [19]. Magmatic zircons of ash layers in the overlying glacial Nantuo Fm. have been dated between $636.3 \pm 4.9 \mathrm{Ma}$ (SHRIMP U-Pb age [19]) and $635.2 \pm 0.6$ (ID TIMS U-Pb age [20]). The Nanhua Basin is a restricted basin that developed on the southwest to northeast striking passive continental margin of the Yangtze Platform that was occasionally connected to the open ocean during the Cryogenian Period [21]. Extensive sedimentological and geochemical data have been obtained from shales of the Datangpo Fm. including iron speciation, trace metal, and rare earth and yttrium (REY) abundances as well as stable C, N, S, Fe, and Mo isotope compositions [10,11,21-26]. The combined geochemical studies suggest that the interglacial Nanhua Basin environment is best described by a stratified water column with anoxic and limited euxinic deep waters. However, deep waters temporarily changed to suboxic conditions associated with widespread dissolution of Mn-oxy/hydroxides (positive Ce anomalies [24]) being deposited on the basin floor which subsequently were transformed into diagenetic Mn carbonates under reducing pore water conditions [22,23]. Reducing pore waters further resulted in a near-quantitative Fe-oxy/hydroxide transformation into sulphides leading to relatively heavier Fe isotope compositions in the remaining seawater [11]. Additionally, a large, dissolved organic carbon pool existed shortly after thawing of the Tiesi'ao glaciers along with high denitrification rates that prevailed throughout the low oxygen conditions of the Nanhua Basin's deep waters $[25,26]$. Molybdenum isotopes of Datangpo Fm. shales show uniformly high $\delta^{98}$ Mo values in the lower part and combined low and variable $\delta^{98}$ Mo values in the upper part, respectively, suggesting euxinic seawater conditions in the lower part of the formation and variable redox conditions in the stratigraphically upper units [21]. 


\section{2. (Bio)Geochemical Cycling of Trace Elements in Precambrian Oceans}

The abundance and availability of trace elements in the Precambrian oceans were greatly different from those of Phanerozoic and modern oceans [27]. Factors controlling the trace elemental budget in the oceans are their local source inputs (riverine, aeolian, benthic flow, and hydrothermal influx), ocean mixing time, and element valency, controlling the elements' chemical behaviour and their availability to form complexes with inorganic or organic ligands or their adsorption onto particle surfaces (cf. [28]).

While $\mathrm{Ni}, \mathrm{Zn}$, and $\mathrm{Cd}$ are commonly present as divalent cations in seawater and form complexes with chloride $\left(\mathrm{Cl}^{-}\right)$, carbonate $\left(\mathrm{CO}_{3}{ }^{2-}\right)$, or oxi/hydroxides $\left(\mathrm{OH}^{-}\right)$, other transition metals such as $\mathrm{U}, \mathrm{Fe}$, $\mathrm{V}, \mathrm{Mn}, \mathrm{Mo}, \mathrm{Co}, \mathrm{Cr}$, and Se exist in a variety of oxidation states which influence their (bio)geochemical cycling in the oceans by variable reaction rates, i.e., their partitioning into authigenic phases such as sulphides, carbonates, or oxi/hydroxides or their availability for complexation with organic ligands [28]. The concept of mean ocean residence time (MORT) includes all processes involved in the modern ocean trace element cycling as the residence time of an element, i.e., the average time from source to sink, depending on how fast the element can be adsorbed on, or incorporated into, a phase that is available for sedimentary deposition [29]. However, in the Precambrian oceans the MORT of most of the elements is unknown and availability of metals and coincident (bio)geochemical cycling may have been very different from modern environments [30-32]. During the Proterozoic and Archean, the oceans had different source contributions [33,34] and the Precambrian oceans were most likely stratified in terms of their oxygen availability with an oxidized surface layer and anoxic to euxinic (containing free $\mathrm{H}_{2} \mathrm{~S}$ ) deep waters [12,35].

Pristine, i.e., nondiagenetic overprinted, nonbiogenic carbonates are believed to be reliable archives for the geochemical compositions of the ambient fluid they precipitate from ([36]). In a fully oxygenated modern ocean, the ability of phototrophic organisms to take up trace elements using special transport enzymes generally causes a relative depletion of enzymatically required trace elements in the photic surface ocean. Together with a relative enrichment of these trace elements in the deeper water column (due to the oxidative dissolution of sinking dead biomass), this creates a nutrient-like element concentration profile. Under stratified ocean conditions the uptake mechanisms of phototrophic organisms in the surface waters should act in the same direction leaving the upper water column relatively depleted in vital trace elements. However, the deposition and preservation of carbonates and OM under reducing conditions can happen under variable environmental conditions influencing the ability of marine sediments to record pristine geochemical signals of the ambient seawater:

- Manganous condition. Under manganous conditions the dissolution of Mn oxy-hydroxides together with a decreased oxidative OM dissolution may ultimately lead to positive Ce anomalies in shale normalized rare earth element (REE) patterns together with enriched Mn concentrations and negative stable $\mathrm{C}$ isotope compositions in carbonates [37-39]. Authigenic Mn carbonates, i.e., rhodochrosite, form within the sediment body [40].

- Anoxic condition. Under truly anoxic $\left(\mathrm{pO}_{2}<10 \mu \mathrm{mol}\right)$ conditions, $\mathrm{OM}$ will not be recycled anymore and deposited in OM-rich shales showing common enrichments of the redox sensitive elements U and Mo [41,42]. By contrast, carbonates will be generally enriched in Mn and Ce due to the breakdown of Mn-oxides [30]. Further, redox sensitive trace metals, such as U, Re, V, and Mo but also $\mathrm{Cd}$, have been shown to be relatively enriched in authigenic carbonate under reducing and anoxic conditions possibly due to supressed oxidative biogeochemical metal recycling [43].

- Euxinic condition. Under euxinic $\left(\mathrm{O}_{2}<10 \mu \mathrm{mol}\right.$, free $\left.\mathrm{H}_{2} \mathrm{~S}\right)$ conditions in the deep water/pore water space authigenic sulphides (e.g., framboidal pyrite grains) or early-diagenetic sulphides (e.g., idiomorphic pyrite grains) can form, binding crucial trace metals such as $\mathrm{Sn}, \mathrm{Zn}, \mathrm{Cd}, \mathrm{Ni}$, and $\mathrm{Cu}[44-47]$.

To qualify the relative enrichments of the above mentioned elements in OM-rich sediments, the analysed bulk rock element abundances are usually normalized to global crustal rock reference materials $[48,49]$ or to the detrital input from the local hinterland [50]. By analogy, the relative 
enrichment of trace elements in nonbiogenic carbonates has been normalized to abundances in Phanerozoic carbonate reference materials $[37,51]$. These methods have shown to be reliable proxies to assess the relative enrichment of vital elements in marine lithologies and (bio)geochemical cycling of these elements in the ancient ocean. However, these proxies are prone to diagenetic modification, especially in the case of redox sensitive and fluid-mobile elements, which are strongly influenced by intermittent oxidizing conditions in the pore water space, remobilizing trace metals and redistributing them within the sediment body [52]. To achieve a better understanding of the element cycling within the water column and the redistribution within the sediment body a holistic view at the level of each individual phase within a mixed sediment lithology is useful.

\subsection{Status Quo of Cd Isotopes in Marine Lithologies}

Cadmium isotopes in marine environments are a promising new tool for studying (bio)geochemical cycling of micronutrients in the oceans [53-55] and in sedimentary archives, such as Fe-Mn crusts [56], carbonates [57,58], microbialites [30], mudstones, and black shales [57-59]. Cd concentrations in seawater show a nutrient-like behaviour, with surface depletion and deep water enrichments. However, the processes leading to this distinct behaviour of $\mathrm{Cd}$ in modern seawater are yet to be fully understood. Utilization of $\mathrm{Cd}$ by marine photosynthetic organisms is the most favoured explanation, leading to a depletion of the surface water $\mathrm{Cd}$ pool [55]. As a possible driver for the $\mathrm{Cd}$ uptake, Price and Morel (1990) [59] described a new variant of the enzyme carbonic anhydrase (Cd-CA), which is active in the resorption of carbon dioxide during photosynthetic processes. In this enzyme, the usual $\mathrm{Zn}$ atom used for the catalysis is replaced by $\mathrm{Cd}$. However, Cd-CA is only known from a few diatom species [60] which first appeared in the Mesozoic.

Cadmium concentrations in the water column generally vary in the opposite direction to the fractionation of stable $\mathrm{Cd}$ isotopes [53,54]. Thus, Cd-depleted surface waters have isotopically 'heavy' Cd signatures, while deep waters exhibit higher Cd concentrations but 'lighter' isotopic compositions. This is considered to reflect surface removal of light isotopes by marine photosynthetic organisms and their sinking and remineralization in bottom waters $[54,55]$. The isotopic composition of seawater is set by the balance of $\mathrm{Cd}$ inputs (and their isotopic composition) and the fractionation on removal to sedimentary sinks. Utilization of $\mathrm{Cd}$ by phytoplankton may be a major process controlling modern seawater $\mathrm{Cd}$ budgets and creating a depth gradient in $\mathrm{Cd}$ concentrations and isotopic composition $[54,55,59]$. Under incomplete oxidative OM recycling, biological uptake may act as an effective $\mathrm{Cd}$ sink and authigenic minerals may store the ambient seawater $\mathrm{Cd}$ isotope composition. Consequently, stable $\mathrm{Cd}$ isotope compositions show broad variations in ancient sediments that may be linked to variations in paleo productivity and redox state $[30,57,58,61-63]$. Many more processes govern the $\mathrm{Cd}$ isotopic compositions of sediments than in seawater. Therefore, when applying $\mathrm{Cd}$ isotopes in ancient marine sediments, additional fractionation processes with different fractionation factors have to be evaluated very carefully. Besides biological utilization, partitioning into calcite [64] or authigenic sulphides $[45,46]$ and variable uptake under changing salinity $[57,65,66]$ may play a role. Unlike the aforementioned processes, inorganic adsorption onto Mn oxides has been shown experimentally to have no significant isotope fractionation at long time scales $(>24 \mathrm{~h})$ [67]. This makes Mn oxides, and potentially diagenetic Mn carbonates formed after reductive oxide dissolution (assuming the second order kinetic fractionation factor into carbonates), potential archives for recording the Cd isotope composition of contemporaneous fluids.

We here used a sequential leaching approach targeting the systematics of bioavailable trace metals and $\mathrm{Cd}$ isotopes in various lithologies from the basal Datangpo Fm., Southwest China, to extract organic and inorganic fractions. We applied a slightly modified sequential leaching procedure [68] on five Cryogenian OM-rich marine sediment samples (one black shale and four overlying OM-rich shales with abundant Mn carbonates and sulphide grains) to study the (bio)geochemical metal cycling and $\mathrm{Cd}$ isotope fractionation in the Cryogenian Nanhua Basin seawater. To better understand the vertical 
metal cycling in the ambient seawater by phototropic life, we here also present for the first time Cd isotope compositions in different leaching fractions from Cryogenian mixed lithologies.

\section{Materials and Methods}

\subsection{Geological Sampling Location and Mineralogy}

Samples for this study, were collected from the Cryogenian basal Datangpo Fm. from drill core zk2303, which was drilled by the Geological Team 103 in the Daotuo area in NE Guizhou Province, South China (Figure 1). The Datangpo Fm. conformably overlies diamictites of the Sturtian Tiesi'ao Fm. [69] and hosts a variety of OM-rich sediments such as black shales, mudstones, and Mn carbonates that were deposited in the semi-restricted Nanhua Basin on the SE slope of the Yangtze Platform with periodical exchange to open ocean [70]. The basal Datangpo Fm. comprises $22 \mathrm{~m}$ of OM-rich black shales (members 1 and 3) which are intercalated by a 6-m-thick OM-rich manganese carbonate bearing layer (member 2) (Figure 1). The black shales are stratigraphically followed by sandy shales of the middle Datangpo Fm. (member 4) and grey siltstones of the upper member 5 (not shown in Figure 1) $[25,26]$. The Datangpo Fm. is topped by the diamictites of the Nantuo Fm. indicative for the start of the Marinoan ice age [2].

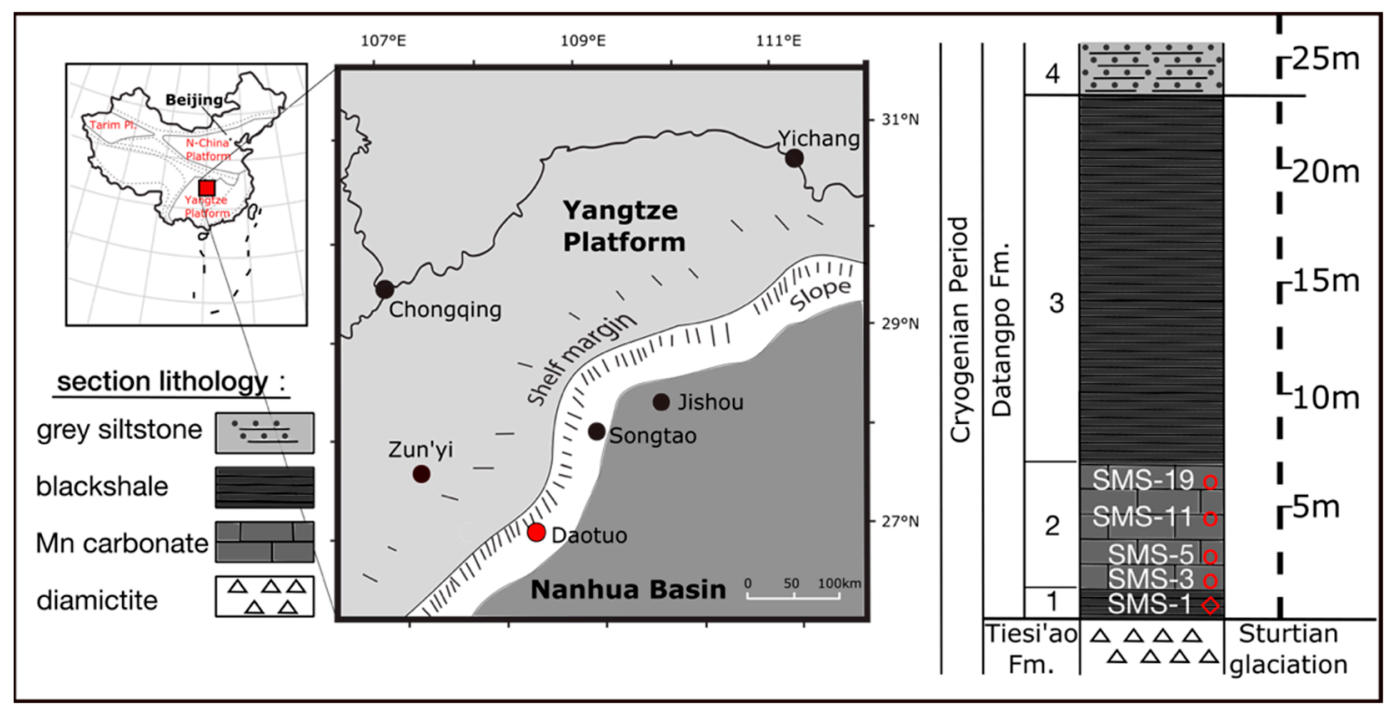

Figure 1. Paleo-geographic map of drill core locality near Daotuo, Northern Guizhou, S China, modified after Jiang et al. (2011) [70]. Stratigraphic column from Wei et al. (2016) [26]. Mn carbonate samples marked in stratigraphic profile as red circles, black shale sample is marked as a red diamond.

In this study, leachates of five representative samples of the lower Datangpo Fm. were analysed. The samples consisted of four OM-rich shales hosting abundant Mn-rich carbonate layers (member 2) that are overlying one black shale sample of member 1. Overall, 30 analytes derived from 5 individual leaching steps of five samples and one duplicate were measured for their trace metal systematics and $\mathrm{Cd}$ isotope compositions. The black shale at the base of the Datangpo Fm. bore detrital minerals, OM, and authigenic sulphides, while scanning electron backscatter diffraction microscopy (EBSD) together with energy-dispersive X-ray spectroscopy (EDX) performed on a JEOL JXA-8230 equipped with an Oxford X-MaxN 20 IE250 at the State Key Laboratory of Marine Geology, School of Ocean and Earth Sciences, Tongji University, Shanghai, and by Xiao et al. (2017) [24] on the OM-rich Mn carbonates obtained rhodochrosite and calico-rhodochrosite minerals, detrital quartz, clays, and feldspar as well as framboidal pyrites of $\sim 10 \mu \mathrm{m}$ diameter. Manganese carbonate minerals were elongated and showed two generations with globular shaped minerals ( $\sim 5 \mu$ diameter) with high Mn content ( $>25$ wt. $\%)$ in the centre surrounded by diffuse Mn beds with Mn contents <25 wt.\% (see Supplementary Materials 
Figure S1). These Mn carbonates are closely associated to framboidal sulphides with individual grain sized between 5-10 $\mu \mathrm{m}$ (see S1) and, less commonly, idiomorphic bigger sulphide grains $(\sim 30 \mu \mathrm{m})$.

\subsection{Sequential Leaching Protocol for Trace Metal Extraction from OM and Mn Carbonate-Rich Shales}

OM-rich shales deposited in restricted basin environments host a variety of different authigenic and detrital phases. The authigenic phases comprise carbonates and OM, leachable in strong alkaline solutions [71], as well as hydroxides, sulphates, and authigenic sulphides. The detrital phases comprise silicate minerals and colloids (such as ashes, dust particles, etc.). For the geochemical analyses we chose five bulk powdered samples of approximately $1 \mathrm{~g}$ from a sample suite that has been described in detail by Wei et al. (2016) [26]. We then performed a sequential leaching procedure (Figure 2) modified after Henrique-Pinto et al. (2016) [68] to extract trace metals associated with a variety of host phases within the powdered rocks. This method, in contrast to traditional Aqua Regia/hydrofluoric acid (HF) bulk rock digestion methods, allows a sequential extraction of moderately volatile elements at low temperature that are bound to organic or colloidal phases and usually provides a better recovery than the total digestion methods of organic rich rocks [68]. Further, sequential leaching methods gave us the possibility to study fractionation of trace metals and its isotopes between different authigenic phases rather than studying bulk rock geochemical signatures that may be corroborated by signals from local detrital sedimentation background into a restricted basin environment.

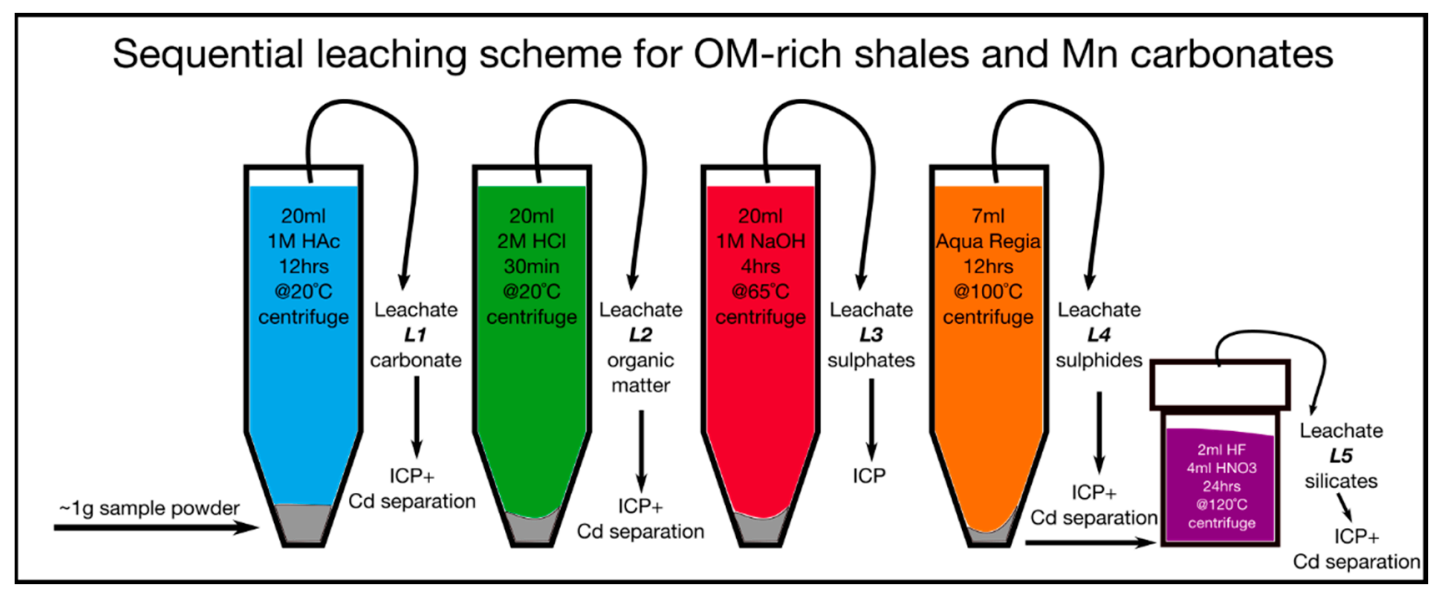

Figure 2. Schematic leaching procedure for OM-rich marine sediments used to obtain reliable trace metal and $\mathrm{Cd}$ isotope compositions in five sequential leachate steps performed on $\mathrm{OM}$ and $\mathrm{Mn}$ carbonate-rich lithologies, modified after Henrique-Pinto et al. (2016) [68].

Step 1: Twenty $\mathrm{mL}$ of $1 \mathrm{M}$ double distilled HAc was added to the sample powders in $50 \mathrm{~mL}$ centrifuge vials, the solution was stirred for $30 \mathrm{~min}$ and reacted overnight. Then the samples were placed in an ultrasonic bath for $30 \mathrm{~min}$, centrifuged at $3500 \mathrm{rpm}$ for $15 \mathrm{~min}$, and finally the leachate L1 was pipetted out and dried down. The residual solids of step 1 remained in the vials.

Step 2: Residual solids of step 1 were treated with $20 \mathrm{~mL} 2 \mathrm{M}$ double distilled $\mathrm{HCl}$, stirred for $30 \mathrm{~min}$, and placed in an ultrasonic bath for $15 \mathrm{~min}$. The leachate L2 then was centrifuged at 3500 rpm for $15 \mathrm{~min}$, pipetted, and dried down. The residual solids of step 2 remained in the vials.

Step 3: Twenty mL 1 M Suprapur ${ }^{\circledR} \mathrm{NaOH}$ were added to the residual solids of step 2. The solution was then stirred for $30 \mathrm{~min}$ and reacted for $4 \mathrm{hrs}$ in a $60{ }^{\circ} \mathrm{C}$ water bath. After placing the solution in an ultrasonic bath for $15 \mathrm{~min}$, it was then centrifuged at $3500 \mathrm{rpm}$ for $15 \mathrm{~min}$. Finally, the leachate L3 was pipetted and dried down. The residual solids of step 3 remained in the c vials.

Step 4: The residual solids of step 3 were treated with $7 \mathrm{~mL}$ Aqua Regia (4 mL conc. double distilled $\mathrm{HCl}$ and $3 \mathrm{~mL}$ conc. double distilled $\mathrm{HNO}_{3}$ ). The solution reacted for 24 hrs before the 
reaction vessels were placed in a water bath at $100{ }^{\circ} \mathrm{C}$ with slightly opened caps. Then, the leachate L4 was diluted with $20 \mathrm{~mL}$ Milli-Q, centrifuged at $3500 \mathrm{rpm}$ for $15 \mathrm{~min}$, pipetted, and dried down. The residual solids of step 4 remained in the vials.

Step 5: In the final step the residual solids of step 4 were transferred into perfluoroalkoxy alkane (PFA) beakers and reacted with 2 steps of $5 \mathrm{~mL}$ of conc. double distilled HF and $1 \mathrm{~mL}$ conc. double distilled $\mathrm{HNO}_{3}$, heated to $120^{\circ} \mathrm{C}$ for $24 \mathrm{hrs}$ with closed caps, and dried down afterwards. To break down the remaining fluorides, the leachate (full digestion of remaining silicates) L5 was treated with repeated dry-down steps of small volumes of conc. $\mathrm{HNO}_{3}$.

Finally, all dried down sequential leachates L1 to L5 were redissolved in double distilled 3\% $\mathrm{HNO}_{3}$ and an internal $500 \mathrm{ppb}$ Rh standard was added. The solutions were analysed on a Thermo Finnigan ICP-MS Element 2, using internal standardization and external calibration methods. We obtained concentrations of potentially bioavailable and partly redox-sensitive trace metals $\mathrm{Co}, \mathrm{Zn}, \mathrm{Ba}$, $\mathrm{V}, \mathrm{Cr}, \mathrm{Mo}, \mathrm{U}, \mathrm{Ni}, \mathrm{Cu}, \mathrm{Mn}$, and $\mathrm{Cd}$ as well as conservative behaving trace metals such as $\mathrm{Zr}, \mathrm{Ti}, \mathrm{Sr}, \mathrm{Nb}$, and Th. Limits of detection (LOD) were calculated as the signal of the analytical blank $+3 x$ standard deviation (SD) of the blank. Repeated measurements of bulk digestions of the GBW07107 (GSR-5) and the USGS SDO-1 shale reference materials proved the methods' analytical accuracy was between $5 \%$ and $30 \%$ relative standard deviation (RSD) (see bottom of Table 1 ) relative to the concentrations reported in Govindaraju (1994) [72]. However, for V and Th distinct deviations (>50\%) from the reference values were observed. Cd concentrations obtained in bulk analyses of the GSR-5 standard were $92 \%$ accurate compared to compiled literature values, whereas its concentrations obtained in bulk analyses of the certified standard reference material (SRM) SDO-1 were about 70\% too high. Therefore, we obtained more precise $\mathrm{Cd}$ concentrations by isotope dilution (ID) and from here on only Cd ID concentration data is discussed. A linear correlation between $\mathrm{Cd}$ concentrations obtained by ICP-MS standard calibration and ID TIMS measurements was obtained, with Cd concentrations obtained by ICP-MS being in average 37\% higher (Supplementary Materials Figure S4). 
Table 1. Trace element concentrations obtained in 5 leachates and shale reference materials.

\begin{tabular}{|c|c|c|c|c|c|c|c|c|c|c|c|c|c|}
\hline Sample ID & Depth (m) & Lithology & Leach Sample & Reagent & $\begin{array}{c}\text { Leached } \\
\text { Phase }\end{array}$ & $\begin{array}{c}\mathrm{Zr} \\
(\mu \mathrm{g} / \mathrm{g})\end{array}$ & $\begin{array}{c}\text { Co } \\
(\mu \mathrm{g} / \mathrm{g})\end{array}$ & $\begin{array}{c}\mathrm{Zn} \\
(\mu \mathrm{g} / \mathrm{g})\end{array}$ & $\begin{array}{c}\mathrm{Ba} \\
(\mu \mathrm{g} / \mathrm{g})\end{array}$ & $\begin{array}{c}\mathrm{V} \\
(\mu \mathrm{g} / \mathrm{g})\end{array}$ & $\begin{array}{c}\mathrm{Cr} \\
(\mu \mathrm{g} / \mathrm{g})\end{array}$ & $\begin{array}{c}\text { Mo } \\
(\mu \mathrm{g} / \mathrm{g})\end{array}$ & $\begin{array}{c}\mathrm{U} \\
(\mu \mathrm{g} / \mathrm{g})\end{array}$ \\
\hline SMS-1 & 1481.3 & black shale & L1.1 & $1 \mathrm{M} \mathrm{HAc}$ & carbonate & LOD & 6.1 & 9.9 & 6.1 & 1.4 & 0.91 & 0.42 & 0.06 \\
\hline SMS-3 & 1480 & $\begin{array}{l}\text { OM-rich Mn } \\
\text { carbonate } \\
\text { OM-rich Mn }\end{array}$ & L1.3 & $1 \mathrm{M} \mathrm{HAc}$ & carbonate & LOD & 8.3 & 12 & 7.2 & 3.0 & 1.7 & 0.67 & 0.02 \\
\hline SMS-5 & 1479.3 & $\begin{array}{c}\text { carbonate } \\
\text { OM-rich Mn }\end{array}$ & L1.5 & $1 \mathrm{M} \mathrm{HAc}$ & carbonate & LOD & 17 & 21 & 3.8 & 1.6 & 0.42 & 1.3 & 0.08 \\
\hline SMS-11 & 1477.3 & $\begin{array}{c}\text { carbonate } \\
\text { OM-rich Mn }\end{array}$ & L1.11 & $1 \mathrm{M} \mathrm{HAc}$ & carbonate & LOD & 1.2 & 4.6 & 11 & 3.4 & 1.4 & 0.26 & LOD \\
\hline SMS-19 & 1475.1 & carbonate & L1.19 & $1 \mathrm{M} \mathrm{HAc}$ & carbonate & LOD & 0.9 & 4.7 & 11.0 & 5.3 & 2.1 & 0.30 & 0.063 \\
\hline SMS-1 & 1481.3 & black shale & $\mathrm{L} 2.1$ & $2 \mathrm{M} \mathrm{HCl}$ & $\mathrm{OM}$ & LOD & 2.6 & 17 & 4.2 & 3.0 & 1.7 & 4.3 & LOD \\
\hline SMS-3 & 1480 & $\begin{array}{l}\text { OM-rich Mn } \\
\text { carbonate } \\
\text { OM-rich Mn }\end{array}$ & L2.3 & $2 \mathrm{M} \mathrm{HCl}$ & $\mathrm{OM}$ & LOD & 4.6 & 40 & 42 & 4.3 & 2.1 & 7.7 & 0.094 \\
\hline SMS-5 & 1479.3 & $\begin{array}{l}\text { carbonate } \\
\text { OM-rich Mn }\end{array}$ & L2.5 & $2 \mathrm{M} \mathrm{HCl}$ & $\mathrm{OM}$ & LOD & 4 & 15 & 174 & 2.0 & 0.68 & 8.6 & LOD \\
\hline SMS-11 & 1477.3 & $\begin{array}{l}\text { carbonate } \\
\text { OM-rich Mn }\end{array}$ & L2.11 & $2 \mathrm{M} \mathrm{HCl}$ & $\mathrm{OM}$ & LOD & 0.65 & 21 & 62 & 1.9 & 1.6 & 2.6 & 0.098 \\
\hline SMS-19 & 1475.1 & carbonate & L2.19 & $2 \mathrm{M} \mathrm{HCl}$ & $\mathrm{OM}$ & LOD & 0.44 & 25 & 64 & 2.9 & 1.6 & 3.3 & 0.21 \\
\hline SMS-1 & 1481.3 & $\begin{array}{l}\text { black shale } \\
\text { OM-rich Mn }\end{array}$ & L3.1 & $1 \mathrm{M} \mathrm{NaOH}$ & sulphate & 16 & 0.15 & 48 & 1053 & 3.4 & 0.9 & 5.6 & LOD \\
\hline SMS-3 & 1480 & $\begin{array}{l}\text { carbonate } \\
\text { OM-rich Mn }\end{array}$ & L3.3 & $1 \mathrm{M} \mathrm{NaOH}$ & sulphate & 4.8 & 0.16 & 57 & 1156 & 3.2 & 1.7 & 8.0 & LOD \\
\hline SMS-5 & 1479.3 & $\begin{array}{l}\text { carbonate } \\
\text { OM-rich Mn }\end{array}$ & L3.5 & $1 \mathrm{M} \mathrm{NaOH}$ & sulphate & 27 & 0.21 & 67 & 1383 & 2.4 & 1.7 & 15 & LOD \\
\hline SMS-11 & 1477.3 & $\begin{array}{l}\text { carbonate } \\
\text { OM-rich Mn }\end{array}$ & L3.11 & $1 \mathrm{M} \mathrm{NaOH}$ & sulphate & 25 & 0.15 & 65 & 1428 & 1.2 & 1.2 & 2.9 & LOD \\
\hline SMS-19 & 1475.1 & carbonate & L3.19 & $1 \mathrm{M} \mathrm{NaOH}$ & sulphate & 27 & 0.15 & 73 & 1509 & 1.5 & 1.3 & 3.3 & LOD \\
\hline SMS-1 & 1481.3 & $\begin{array}{l}\text { black shale } \\
\text { OM-rich Mn }\end{array}$ & L4.1 & Aqua Regia & sulphide & LOD & 17 & 19 & 34 & 2.2 & 4.4 & 13 & LOD \\
\hline SMS-3 & 1480 & $\begin{array}{l}\text { carbonate } \\
\text { OM-rich Mn }\end{array}$ & L4.3 & Aqua Regia & sulphide & LOD & 17 & 17 & 25 & 2.4 & 6.5 & 15 & 0.016 \\
\hline SMS-5 & 1479.3 & $\begin{array}{l}\text { carbonate } \\
\text { OM-rich Mn }\end{array}$ & L4.5 & Aqua Regia & sulphide & LOD & 17 & 18 & 22 & 2.0 & 1.3 & 19 & LOD \\
\hline SMS-11 & 1477.3 & $\begin{array}{l}\text { carbonate } \\
\text { OM-rich Mn }\end{array}$ & L4.11 & Aqua Regia & sulphide & LOD & 12 & 19 & 15 & 2.3 & 4.9 & 3.7 & LOD \\
\hline SMS-19 & 1475.1 & carbonate & L4.19 & Aqua Regia & sulphide & LOD & 15 & 30 & 16 & 2.6 & 4.6 & 4.8 & 0.026 \\
\hline
\end{tabular}


Table 1. Cont

\begin{tabular}{|c|c|c|c|c|c|c|c|c|c|c|c|c|c|}
\hline SMS-1 & 1481.3 & $\begin{array}{l}\text { black shale } \\
\text { OM-rich Mn }\end{array}$ & L5.1 & $\begin{array}{c}\text { conc. } \\
\text { HF+HNO3 } \\
\text { conc. }\end{array}$ & silicate & 46 & 1.3 & 16 & 311 & 79 & 20 & 1.2 & 0.48 \\
\hline SMS-3 & 1480 & $\begin{array}{l}\text { carbonate } \\
\text { OM-rich Mn }\end{array}$ & L5.3 & $\begin{array}{l}\mathrm{HF}+\mathrm{HNO} 3 \\
\text { conc. }\end{array}$ & silicate & 251 & 5.0 & 71 & 1295 & 359 & 96 & 6.0 & 3.0 \\
\hline SMS-5 & 1479.3 & $\begin{array}{c}\text { carbonate } \\
\text { OM-rich Mn }\end{array}$ & L5.5 & $\begin{array}{l}\mathrm{HF}+\mathrm{HNO} 3 \\
\text { conc. }\end{array}$ & silicate & 71 & 1.5 & 45 & 387 & 107 & 26 & 1.6 & 0.67 \\
\hline SMS-11 & 1477.3 & $\begin{array}{l}\text { carbonate } \\
\text { OM-rich Mn }\end{array}$ & L5.11 & $\begin{array}{l}\mathrm{HF}+\mathrm{HNO} 3 \\
\text { conc. }\end{array}$ & silicate & 890 & 13 & 437 & 6765 & 1020 & 307 & 5.3 & 13 \\
\hline SMS-19 & 1475.1 & carbonate & L5.19 & $\mathrm{HF}+\mathrm{HNO} 3$ & silicate & 101 & 1.9 & 37 & 600 & 121 & 38 & 0.93 & 1.0 \\
\hline \multicolumn{2}{|c|}{$\begin{array}{l}\text { SDO-1.1 } \\
\text { Govindaraju et al., } 1994 \\
\text { accuracy (\%) }\end{array}$} & $\begin{array}{l}\text { OM-rich silt } \\
\text { shale }\end{array}$ & bulk $(n=5)$ & $\begin{array}{c}\mathrm{HF} / \mathrm{HNO} 3 \\
\text { bomb }\end{array}$ & $\begin{array}{c}\text { total } \\
\text { digest. }\end{array}$ & $\begin{array}{l}195 \\
165 \\
122\end{array}$ & $\begin{array}{c}47 \\
47 \\
109\end{array}$ & $\begin{array}{l}57 \\
64 \\
95\end{array}$ & $\begin{array}{c}396 \\
397 \\
96 \\
\end{array}$ & $\begin{array}{l}172 \\
160 \\
119\end{array}$ & $\begin{array}{c}69 \\
66 \\
116 \\
\end{array}$ & $\begin{array}{l}170 \\
134 \\
127\end{array}$ & $\begin{array}{l}45 \\
49 \\
82\end{array}$ \\
\hline \multicolumn{2}{|c|}{$\begin{array}{l}\text { GBW07107 (GSR-5) } \\
\text { Govindaraju et al., } 1994 \\
\text { accuracy (\%) }\end{array}$} & shale & bulk $(n=6)$ & $\begin{array}{c}\mathrm{HF} / \mathrm{HNO} 3 \\
\text { bomb }\end{array}$ & $\begin{array}{c}\text { total } \\
\text { digest. }\end{array}$ & $\begin{array}{c}120 \\
96 \\
125\end{array}$ & $\begin{array}{c}24 \\
21 \\
117\end{array}$ & $\begin{array}{c}61 \\
50 \\
122\end{array}$ & $\begin{array}{c}406 \\
450 \\
90\end{array}$ & $\begin{array}{c}130 \\
87 \\
150\end{array}$ & $\begin{array}{c}131 \\
99 \\
133 \\
\end{array}$ & $\begin{array}{l}0.5 \\
0.4 \\
147\end{array}$ & $\begin{array}{l}1.3 \\
1.5 \\
86\end{array}$ \\
\hline \multicolumn{14}{|c|}{ LOD $=$ below detection limit (blank $+3 x$ standard deviation of blank) } \\
\hline Sample ID & Depth (m) & Lithology & Leach Sample & Reagent & $\begin{array}{l}\text { Leached } \\
\text { Phase }\end{array}$ & $\begin{array}{c}\mathrm{Ni} \\
(\mu \mathrm{g} / \mathrm{g})\end{array}$ & $\begin{array}{c}\mathrm{Cu} \\
(\mu \mathrm{g} / \mathrm{g})\end{array}$ & $\begin{array}{c}\text { Mn } \\
(\mu \mathrm{g} / \mathrm{g})\end{array}$ & $\begin{array}{c}\mathrm{Ti} \\
(\mu \mathrm{g} / \mathrm{g})\end{array}$ & $\begin{array}{c}\mathrm{Sr} \\
(\mu \mathrm{g} / \mathrm{g})\end{array}$ & $\begin{array}{c}\mathrm{Nb} \\
(\mu \mathrm{g} / \mathrm{g})\end{array}$ & $\begin{array}{c}\text { Cd } \\
(\mu \mathrm{g} / \mathrm{g})\end{array}$ & $\begin{array}{c}\text { Th } \\
(\mu \mathrm{g} / \mathrm{g})\end{array}$ \\
\hline SMS-1 & 1481.3 & $\begin{array}{l}\text { black shale } \\
\text { OM-rich Mn }\end{array}$ & L1.1 & 1 M HAc & carbonate & 20 & 11 & 676 & 0.51 & 24 & 0.12 & 0.37 & LOD \\
\hline SMS-3 & 1480 & $\begin{array}{c}\text { carbonate } \\
\text { OM-rich Mn }\end{array}$ & L1.3 & $1 \mathrm{M} \mathrm{HAc}$ & carbonate & 24 & 12 & 3811 & LOD & 40 & 0.043 & 0.39 & 0.003 \\
\hline SMS-5 & 1479.3 & $\begin{array}{c}\text { carbonate } \\
\text { OM-rich Mn }\end{array}$ & L1.5 & $1 \mathrm{M} \mathrm{HAc}$ & carbonate & 35 & 21 & 2205 & LOD & 20 & 0.063 & 0.41 & 0.20 \\
\hline SMS-11 & 1477.3 & $\begin{array}{c}\text { carbonate } \\
\text { OM-rich Mn }\end{array}$ & L1.11 & $1 \mathrm{M} \mathrm{HAc}$ & carbonate & 1.7 & 0.41 & 2829 & LOD & 40 & 0.034 & 0.070 & 0.081 \\
\hline SMS-19 & 1475.1 & carbonate & L1.19 & $1 \mathrm{M} \mathrm{HAc}$ & carbonate & 2.2 & 0.19 & 4098 & LOD & 48 & 0.032 & 0.066 & 0.099 \\
\hline SMS-1 & 1481.3 & $\begin{array}{l}\text { black shale } \\
\text { OM-rich Mn }\end{array}$ & L2.1 & $2 \mathrm{M} \mathrm{HCl}$ & $\mathrm{OM}$ & 4.7 & 13 & 117 & 2.8 & 12 & 0.038 & 0.16 & 0.59 \\
\hline SMS-3 & 1480 & $\begin{array}{c}\text { carbonate } \\
\text { OM-rich Mn }\end{array}$ & $\mathrm{L} 2.3$ & $2 \mathrm{M} \mathrm{HCl}$ & $\mathrm{OM}$ & 6.4 & 14 & 407 & 5.7 & 20 & 0.038 & 0.30 & 1.0 \\
\hline SMS-5 & 1479.3 & $\begin{array}{c}\text { carbonate } \\
\text { OM-rich Mn }\end{array}$ & L2.5 & $2 \mathrm{M} \mathrm{HCl}$ & $\mathrm{OM}$ & 4 & 11 & 224 & 0.67 & 29 & 0.046 & 0.082 & 2.3 \\
\hline SMS-11 & 1477.3 & $\begin{array}{l}\text { carbonate } \\
\text { OM-rich Mn }\end{array}$ & L2.11 & $2 \mathrm{M} \mathrm{HCl}$ & $\mathrm{OM}$ & 2.4 & 2.5 & 234 & 7.1 & 1.8 & 0.038 & 0.092 & 1.1 \\
\hline SMS-19 & 1475.1 & carbonate & L2.19 & $2 \mathrm{M} \mathrm{HCl}$ & $\mathrm{OM}$ & 1.9 & 2.7 & 319 & 10 & 11 & 0.038 & 0.099 & 1.3 \\
\hline
\end{tabular}


Table 1. Cont.

\begin{tabular}{|c|c|c|c|c|c|c|c|c|c|c|c|c|c|}
\hline SMS-1 & 1481.3 & black shale & L3.1 & $1 \mathrm{M} \mathrm{NaOH}$ & sulphate & LOD & 0.17 & 11 & 5.3 & 12 & 0.090 & 0.035 & LOD \\
\hline SMS-3 & 1480 & $\begin{array}{l}\text { OM-rich Mn } \\
\text { carbonate } \\
\text { OM-rich Mn }\end{array}$ & L3.3 & $1 \mathrm{M} \mathrm{NaOH}$ & sulphate & LOD & 0.20 & 7.0 & 2.6 & 9.6 & 0.059 & 0.002 & LOD \\
\hline SMS-5 & 1479.3 & $\begin{array}{l}\text { carbonate } \\
\text { OM-rich Mn }\end{array}$ & L3.5 & $1 \mathrm{M} \mathrm{NaOH}$ & sulphate & LOD & 0.48 & 13 & 6.5 & 17 & 0.11 & 0.065 & LOD \\
\hline SMS-11 & 1477.3 & $\begin{array}{c}\text { carbonate } \\
\text { OM-rich Mn }\end{array}$ & L3.11 & $1 \mathrm{M} \mathrm{NaOH}$ & sulphate & LOD & 0.26 & 7.8 & 5.6 & 16 & 0.11 & 0.020 & LOD \\
\hline SMS-19 & 1475.1 & carbonate & L3.19 & $1 \mathrm{M} \mathrm{NaOH}$ & sulphate & LOD & 0.29 & 7.0 & 6.2 & 17 & 0.11 & 0.016 & LOD \\
\hline SMS-1 & 1481.3 & black shale & L4.1 & Aqua Regia & sulphide & 21 & 28 & 256 & 12 & 3.7 & 0.12 & 0.23 & 1.1 \\
\hline SMS-3 & 1480 & $\begin{array}{l}\text { OM-rich Mn } \\
\text { carbonate } \\
\text { OM-rich Mn }\end{array}$ & L4.3 & Aqua Regia & sulphide & 22 & 25 & 392 & 7.6 & 4.4 & 0.075 & 0.25 & 1.6 \\
\hline SMS-5 & 1479.3 & $\begin{array}{c}\text { carbonate } \\
\text { OM-rich Mn }\end{array}$ & L4.5 & Aqua Regia & sulphide & 17 & 24 & 309 & 3.9 & 6.3 & 0.046 & 0.22 & 1.1 \\
\hline SMS-11 & 1477.3 & $\begin{array}{l}\text { carbonate } \\
\text { OM-rich Mn }\end{array}$ & L4.11 & Aqua Regia & sulphide & 24 & 25 & 312 & 5.6 & 2.0 & 0.060 & 0.23 & 2.0 \\
\hline SMS-19 & 1475.1 & carbonate & L4.19 & Aqua Regia & sulphide & 27 & 27 & 372 & 5.9 & 2.2 & 0.060 & 0.27 & 2.6 \\
\hline SMS-1 & 1481.3 & $\begin{array}{l}\text { black shale } \\
\text { OM-rich Mn }\end{array}$ & L5.1 & $\begin{array}{l}\text { conc. } \\
\mathrm{HF}+\mathrm{HNO} 3 \\
\text { conc. }\end{array}$ & silicate & 0.82 & 2.8 & 35 & 1114 & 5.0 & 1.7 & 0.025 & LOD \\
\hline SMS-3 & 1480 & $\begin{array}{l}\text { carbonate } \\
\text { OM-rich Mn }\end{array}$ & L5.3 & $\begin{array}{l}\mathrm{HF}+\mathrm{HNO} 3 \\
\text { conc. }\end{array}$ & silicate & 8.0 & 12 & 139 & 5855 & 19 & 6.7 & 0.24 & 0.86 \\
\hline SMS-5 & 1479.3 & $\begin{array}{c}\text { carbonate } \\
\text { OM-rich Mn }\end{array}$ & L5.5 & $\begin{array}{l}\mathrm{HF}+\mathrm{HNO} 3 \\
\text { conc. }\end{array}$ & silicate & 0.45 & 2.8 & 56 & 1167 & 9.5 & 1.5 & 0.047 & LOD \\
\hline SMS-11 & 1477.3 & $\begin{array}{l}\text { carbonate } \\
\text { OM-rich Mn }\end{array}$ & L5.11 & $\begin{array}{l}\mathrm{HF}+\mathrm{HNO} 3 \\
\text { conc. }\end{array}$ & silicate & 28 & 38 & 585 & 17049 & 88 & 8.9 & 0.82 & 7.9 \\
\hline SMS-19 & 1475.1 & carbonate & L5.19 & $\mathrm{HF}+\mathrm{HNO} 3$ & silicate & 2.6 & 4.1 & 87 & 2773 & 6.9 & 9.8 & 0.084 & LOD \\
\hline \multicolumn{2}{|c|}{$\begin{array}{l}\text { Govindaraju et al., } 1994 \\
\text { accuracy (\%) }\end{array}$} & $\begin{array}{l}\text { OM-rich silt } \\
\text { shale }\end{array}$ & bulk $(n=5)$ & $\begin{array}{l}\mathrm{HF} / \mathrm{HNO} 3 \\
\text { bomb }\end{array}$ & $\begin{array}{c}\text { total } \\
\text { digest. }\end{array}$ & $\begin{array}{l}107 \\
100 \\
108\end{array}$ & $\begin{array}{l}52 \\
60 \\
87 \\
\end{array}$ & $\begin{array}{l}313 \\
300 \\
104 \\
\end{array}$ & $\begin{array}{r}4506 \\
4260 \\
106 \\
\end{array}$ & $\begin{array}{c}87 \\
75 \\
116 \\
\end{array}$ & $\begin{array}{c}15 \\
11 \\
127 \\
\end{array}$ & $\begin{array}{l}0.51 \\
0.30 \\
170 \\
\end{array}$ & $\begin{array}{l}5.9 \\
10 \\
59\end{array}$ \\
\hline \multicolumn{2}{|c|}{$\begin{array}{l}\text { GBW07107 (GSR-5) } \\
\text { Govindaraju et al., } 1994 \\
\text { accuracy (\%) }\end{array}$} & shale & bulk $(n=6)$ & $\begin{array}{l}\mathrm{HF} / \mathrm{HNO} 3 \\
\text { bomb }\end{array}$ & $\begin{array}{c}\text { total } \\
\text { digest. }\end{array}$ & $\begin{array}{c}45 \\
37 \\
123\end{array}$ & $\begin{array}{c}42 \\
42 \\
101\end{array}$ & $\begin{array}{l}180 \\
173 \\
104\end{array}$ & $\begin{array}{c}1158 \\
4719 \\
25\end{array}$ & $\begin{array}{c}103 \\
90 \\
115\end{array}$ & $\begin{array}{c}15 \\
14 \\
102\end{array}$ & $\begin{array}{c}0.030 \\
0.033 \\
92\end{array}$ & $\begin{array}{l}7.0 \\
13 \\
54\end{array}$ \\
\hline
\end{tabular}




\subsection{Stable Cd Isotope Analysis}

Cadmium stable isotope analyses were performed after adequate $\mathrm{Cd}$ purification from four leaching steps, L1, L2, L4, and L5 (total Cd concentrations in L3 were below $5 \mathrm{ng}$ and, therefore, below our detection limit for accurate TIMS analyses). Therefore, we used aliquots containing approximately 50-100 ng of Cd mixed with an optimal amount of a ${ }^{106} \mathrm{Cd}-{ }^{108} \mathrm{Cd}$ double spike solution, [62] dried, and re-equilibrated the mixture in ultrapure $0.5 \mathrm{M} \mathrm{HBr}$. The ${ }^{106} \mathrm{Cd}-{ }^{108} \mathrm{Cd}$ double spike was originally calibrated against an in-house JMC Cd Plasma solution (Lot: 15922032) at the Max-Planck-Institut für Chemie in Mainz, assuming ${ }^{110} \mathrm{Cd} /{ }^{112} \mathrm{Cd}=0.520089$ [73] for unspiked cadmium. The solutions were then loaded onto BioRad ${ }^{\circledR}$ Polyprep columns filled with $200 \mu \mathrm{L}$ AG1-X8 anion-exchange resin (100-200 mesh) to retain the Cd. The resin was rinsed repeatedly with $1 \mathrm{~N} \mathrm{HCl}$ (to remove traces of bromide and matrix) followed by elution of the $\mathrm{Cd}$ with $0.25 \mathrm{~N} \mathrm{HNO}_{3}$. For further purification, the eluted $\mathrm{Cd}$ was acidified to $\sim 0.5 \mathrm{~N} \mathrm{HBr}$ and passed a second time through the columns in order to achieve suitable yields of $\mathrm{Cd}$. Then, 5-50 ng of $\mathrm{Cd}$ were loaded onto zone refined degassed $\mathrm{Re}$ filaments and measured at $\sim 150{ }^{\circ} \mathrm{C}$ in static multi-collection mode on a Thermo Scientific Triton Thermal Ionisation Mass Spectrometer (TIMS) at the Max-Planck-Institut für Chemie in Mainz. The data reduction was achieved by using an in-house coded double spike algorithm assuming exponential natural and instrumental fractionation law. Our procedural blank for the Cd chemistry was $120 \mathrm{pg} / \mathrm{g}$, obtained by isotope dilution and was insignificant compared to $\mathrm{Cd}$ concentrations being at least 50 to 500 times higher in the samples. Following [74], variations in the $\mathrm{Cd}$ isotope compositions are expressed as $\varepsilon^{112 / 110} \mathrm{Cd}$ values (deviations of ${ }^{112} \mathrm{Cd} /{ }^{110} \mathrm{Cd}$ in parts per 10,000 from the zero reference material (RM) of the National Institute of Standardisation (NIST) SRM 3108:

$$
\varepsilon^{112 / 110} C d=\left(\frac{112_{C d} / 110_{C d s a m p l e}}{112_{C d} / 110_{C d R M}}-1\right) \times 10^{4}
$$

Long-term reproducibility of spiked NIST SRM $3108 \mathrm{Cd}$ isotope measurements at MPI Mainz is $0.4 \varepsilon^{112 / 110} \mathrm{Cd}$ units.

\subsection{Stable $C$ and $O$ Isotope Analyses in Carbonate}

Stable $\mathrm{O}$ and $\mathrm{C}$ isotope composition analyses in the phosphoric acid leachable carbonate fraction followed the protocol described in [75]. Therefore, approximately $100 \mu \mathrm{g}$ of carbonate powder were reacted with $30 \mu \mathrm{L}$ of $100 \%$ phosphoric acid at $70{ }^{\circ} \mathrm{C}$ for ca. $1.5 \mathrm{~h}$. The $\mathrm{O}$ and $\mathrm{C}$ isotope ratios were then analysed in the liberated $\mathrm{CO}_{2}$ gas using a Thermo Finnigan Gasbench II coupled with a Thermo Scientific MAT-253 mass spectrometer at the State Key Laboratory for Mineral Deposits Research at Nanjing University. As a reference gas, $\mathrm{CO}_{2}$ calibrated against the Vienna Pee Dee Belemnite (V-PDB) standard was used. The reported isotope ratios were all given in the delta notation, as per mill deviation from the V-PDB standard [76]. The analytical precision, based on the reproducibility of our in-house $\mathrm{CaCO}_{3}$ carbonate standard reference material (SRM) GBW04405, was better than $0.1 \%$ o (standard deviation, 1SD) for both $\mathrm{O}$ and $\mathrm{C}$ isotopes.

\section{Results}

\subsection{Trace Metal Concentrations of Sequential Leachates}

Trace element concentrations of five different leaching steps from the studied lithologies of the basal Datangpo Fm. showed little variation between each sample but varied systematically between the performed leaching steps and between Mn carbonate and shale lithologies (Table 1, Figure 3A). We here report the absolute obtained concentrations of trace metals that were considered important in supporting phototrophic life: $\mathrm{Cd}, \mathrm{Co}, \mathrm{Cu}, \mathrm{Ni}, \mathrm{Zn}, \mathrm{Ba}, \mathrm{Mo}, \mathrm{Ba}$, and $\mathrm{Mn}$ [28] (Figure 3A). These concentrations were obtained on five sequential leaching steps (L1 to L5) and the relative distribution of each element between the different leachates is given in Figure 3B. Finally, relative concentrations 
of the bioavailable metals in L1, L2, L3, and L4 were calculated relative to concentrations in L5 (total digestion of remaining detrital silicates) representing the detrital nondissolved weathering input into the basin (Figure 3C). The equation used to normalize the relative trace element concentration in each leachate to L5 is given below:

$$
\text { norm.tr. metal }=\frac{\text { conc. } L i_{\text {tr. metal }}}{\text { conc. } L 5_{\text {tr. metal }}}
$$

with $i=1$ to 4 .
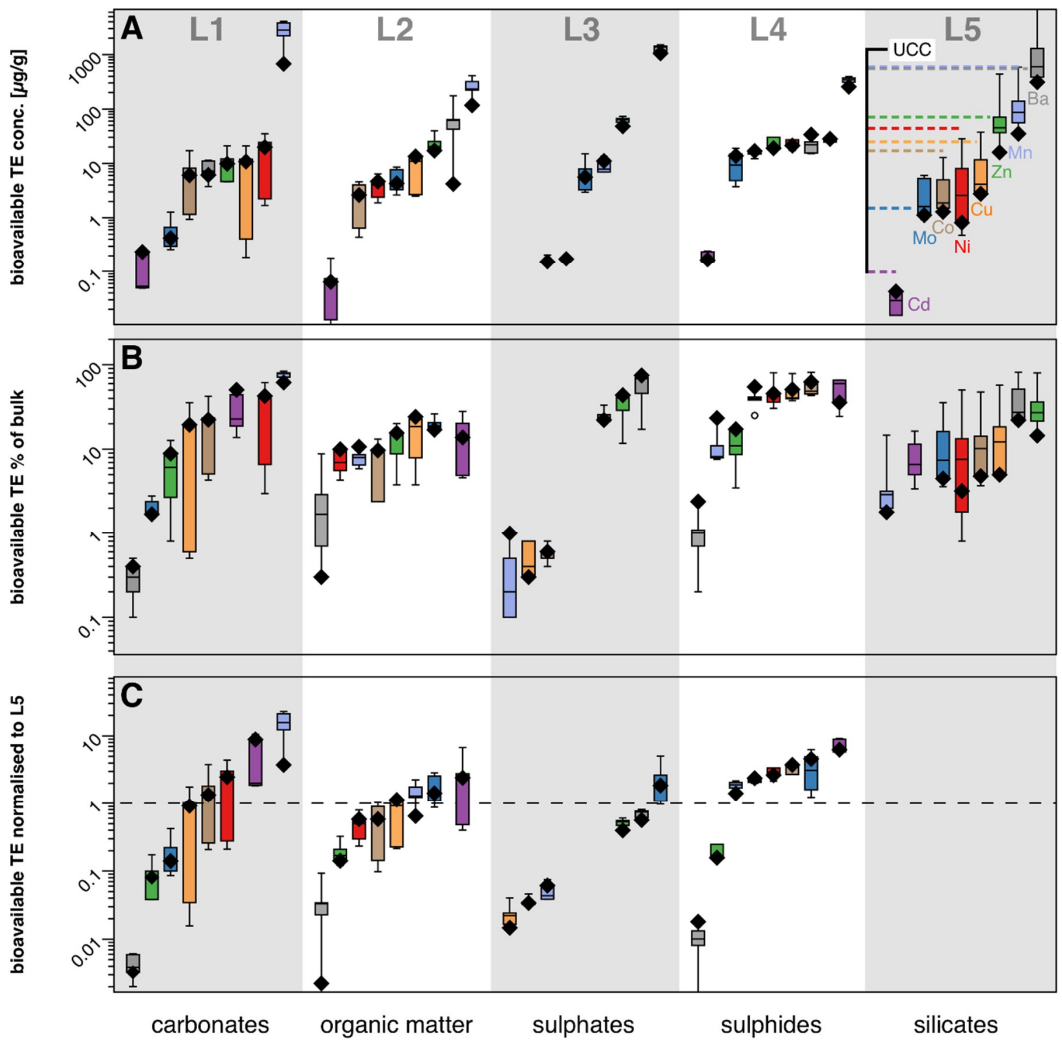

Figure 3. (A) Whisker plots of obtained bioavailable metal concentrations on five different leachates (L1, L2, L3, L4, L5) from mixed lithologies, relative to the respective mineral/authigenic phases being leached. The diamonds represent the composition of the black shale sample SMS- 1 studied. The dashed lines in L5 represent average upper continental crust (UCC) concentrations of the respective elements [77]; all whiskers are sorted to ascending median values, colours refer to elements as obtained in L5. (B) Whisker plots of obtained bioavailable metal concentrations on five different leachates (L1, L2, L3, L4, L5) from mixed lithologies, as percentage of the bulk samples concentration of the respective element. (C) Whisker plots of obtained bioavailable metal concentrations on four different leachates (L1, L2, L3, L4) from mixed lithologies normalized to concentrations obtained in L5 representing a total digestion of the detrital minerals as analogue for the hinterland weathering input of trace metals in the restricted Nanhua Basin.

In leaching step one (L1, leaching of carbonates), average $\mathrm{Cd}$ and Mo concentrations were lower by an order of magnitude compared to $\mathrm{Co}, \mathrm{Ba}, \mathrm{Zn}, \mathrm{Cu}$, and $\mathrm{Ni}$, while $\mathrm{Mn}$ was enriched by three orders of magnitude with an average concentration of $2723 \mu \mathrm{g} / \mathrm{g}( \pm 1373 \mathrm{SD})$ and making up to $84 \%$ of the total Mn budget, which was to be expected for Mn-rich carbonates being leached in L1. Besides the Ti and Mn concentrations, which were five times higher in the Mn carbonates than in the studied shale sample (black diamonds in Figure 3B,C), all other trace element concentrations did not differ between the two lithologies. Cd concentrations in this leachate accounted for $14 \%$ to $51 \%$ of the total Cd budget (Figure 3B). Normalizing the obtained concentrations to the L5 revealed clear relative enrichments of $\mathrm{Cd}, \mathrm{Ni}$, and $\mathrm{Mn}$ in the leached phases, while Ba, Zn, and Mo were relatively depleted (Figure 3C). 
While in leaching step two (L2, leaching of organic matter), Cd, Co, Cu, and Mo as well as Ni showed similar low average concentrations $(0.068 \mu \mathrm{g} / \mathrm{g}( \pm 0.068), 2.4 \mu \mathrm{g} / \mathrm{g}( \pm 1.9), 5.3 \mu \mathrm{g} / \mathrm{g}( \pm 2.7), 8.8 \mu \mathrm{g} / \mathrm{g}$ $( \pm 5.8)$, and $3.9 \mu \mathrm{g} / \mathrm{g}( \pm 21.8)(1 \mathrm{SD})$ ) with Cd concentrations making up $5 \%$ to $28 \%$ of the total Cd budget (Figure 3B). The average concentrations of $\mathrm{Zn}, \mathrm{Ba}$, and Mn were significantly higher $(23.4 \mu \mathrm{g} / \mathrm{g}( \pm 9.8)$, $69.3 \mu \mathrm{g} / \mathrm{g}( \pm 63.3), 2608.8 \mu \mathrm{g} / \mathrm{g}( \pm 109)(1 \mathrm{SD}))$. For most elements no significant variations between lithologies were observed. Ba and Mn showed the lowest concentrations in the shale. Mn and Mo were relatively enriched against the local detrital background in the leached phases while $\mathrm{Zn}$ and $\mathrm{Ba}$ were depleted and $\mathrm{Ni}, \mathrm{Co}, \mathrm{Cd}$, and $\mathrm{Cu}$ showed a ubiquitous enrichment behaviour (Figure 3C).

Leaching Step three (L3, leaching of sulphates) did not yield any Ni concentrations above the detection limit and also average Cd concentrations obtained by ICP-MS were the lowest within these leached phases $(27 \mathrm{ng} / \mathrm{g}( \pm 24,1 \mathrm{SD}))$. Cd ID TIMS data was not obtained on this leachate due to low concentrations. Average Ba concentrations were the highest in this leachate phase due to the dissolution of Ba sulphates with $1306 \mu \mathrm{g} / \mathrm{g}( \pm 5193,1 \mathrm{SD})$. Ba concentration in L2 made up 17\% to 75\% of total Ba budgets (Figure 3B). Concentrations of $\mathrm{Co}$ and $\mathrm{Cu}$ were low while Mn, Mo, and Zn showed higher abundances, with the latter three also revealing detrital background normalized enrichments (Figure 3C). No significant trace metal concentration variations between lithologies were observed.

Leaching step four (L4, leaching of sulphides) revealed relatively high average Cd concentrations of $(0.19 \mu \mathrm{g} / \mathrm{g}( \pm 0.04 .1 \mathrm{SD})$, which accounted for $36 \%$ to $66 \%$ of the total Cd budget (Figure $3 \mathrm{~B})$. However, absolute $\mathrm{Cd}$ concentrations were about two orders of magnitude lower than the concentrations of Mo, $\mathrm{Co}, \mathrm{Zn}, \mathrm{Ni}, \mathrm{Ba}$, and $\mathrm{Cu}$ in the leached phases and up to three orders of magnitude lower than Mn. Concentrations of Ba were extremely low in the shale sample $(310 \mu \mathrm{g} / \mathrm{g})$ but rose to over $6000 \mu \mathrm{g} / \mathrm{g}$ at the top of the Mn carbonates, while Ti concentrations were the highest in the L4 of the shale lithology. Despite $\mathrm{Zn}$ and $\mathrm{Ba}$, all other trace metals were relatively enriched over the local detrital background (Figure 3C).

In leaching step five (L5, total digestion of the remaining detrital silicates) increasing average concentrations were obtained from $\mathrm{Mo}, \mathrm{Co}, \mathrm{Ni}, \mathrm{Cu}, \mathrm{Zn}$, and $\mathrm{Mn}$ to $\mathrm{Ba}$, matching concentrations reported for the average upper continental crust (dashed coloured lines [77]). Average obtained $\mathrm{Cd}$ concentrations for two samples were $(0.03 \mu \mathrm{g} / \mathrm{g}( \pm 0.02,1 \mathrm{SD}))$ denoting for $4 \%$ to $16 \%$ of total $\mathrm{Cd}$ budgets (Figure 3B), which was lower than values reported for the upper continental crust UCC but in the same range as the SRM GSR-5, arguing for the leachate of detrital minerals.

\subsection{Stable $\mathrm{C}, \mathrm{O}$, and $\mathrm{Cd}$ Isotope Compositions}

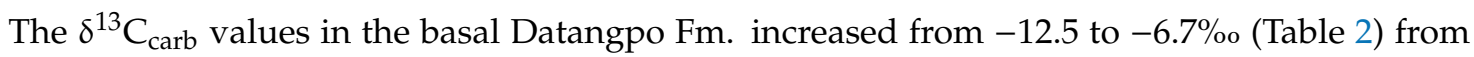
base to top in the studied shale and Mn carbonates. The $\delta^{13} C_{\text {org }}(-31.8$ to $-31.5 \%$ o $)$ values had almost

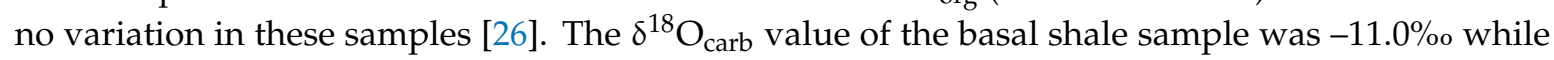
the overlying Mn carbonates were between -9.3 and $-08.4 \%$ o (Table 2 ). We further obtained $\mathrm{Cd}$ isotopic composition in four out of five sequential leaching steps, excluding L3 due to very low Cd concentrations which was not suitable for precise analyses on the TIMS. All Cd isotopic compositions are expressed here as $\varepsilon^{112 / 110} \mathrm{Cd}$ (Equation (1)). The 18 performed analyses $(5 \times \mathrm{L} 1,5 \times \mathrm{L} 2,5 \times \mathrm{L} 3$ +1 duplicate, and $2 \times$ L5) showed a total variation of $7.5 \varepsilon$ units (Table 3 , Figure $3 \mathrm{~A}$ ). Variations in $\varepsilon^{112 / 110} \mathrm{Cd}$ of L1 ranged from -4.3 to +0.66 , with the highest total variation and the heaviest value being recorded in the shale lithology (diamonds in Figure 3A). Values obtained in L2 ranged from +0.13 to +2.9 (again with the heaviest value obtained in the shale). The L 4 values ranged from +0.59 to $+3.2\left(+1.5\right.$ in the shale). Duplicate analyses of one L4 sample obtained $\varepsilon^{112 / 110} \mathrm{Cd}$ values of +0.59 and +0.99 , respectively, (overlapping within the long-term reproducibility of the spiked NIST SRM $3108 \mathrm{Cd}$ isotope measurements of $0.4 \varepsilon^{112 / 110} \mathrm{Cd}$ units). Due to low Cd concentrations in L5, only two reliable stable $\mathrm{Cd}$ isotope compositions were obtained, the shale sample having a $\varepsilon^{112 / 110} \mathrm{Cd}$ of 0.02 and $42.5 \mathrm{ng} / \mathrm{g} \mathrm{Cd}$ and the Mn carbonate sample having a $\varepsilon^{112 / 110} \mathrm{Cd}$ of -0.34 and $15.5 \mathrm{ng} / \mathrm{g} \mathrm{Cd}$. These values overlapped in error with average loess compositions reported in [56] $\left(\varepsilon^{112 / 110} \mathrm{Cd}\right.$ relative to NIST SRM $3108=+0.04 \pm 0.31$ ) as an up-to-date best fit for the composition of the upper continental crust. 
Table 2. $\mathrm{C}$ and $\mathrm{O}$ isotope compositions in carbonate.

\begin{tabular}{|c|c|c|c|c|c|c|c|c|c|}
\hline Sample ID & Lithology & Core Depth (m) & $\delta^{13} C_{\text {carb }}$ & $2 \mathrm{SD}$ & $\delta^{18} \mathrm{O}_{\text {carb }}$ & $2 \mathrm{SD}$ & $\delta^{13} C_{\text {org }}$ & $2 \mathrm{SD}$ & Total Organic Carbon (wt.\%) \\
\hline SMS-1 & black shale & 1481.3 & -12.21 & 0.34 & -11.03 & 0.66 & -31.76 & 0.03 & 3.78 \\
\hline SMS-3 & OM-rich Mn carbonate & 1480 & -12.54 & 0.64 & -9.31 & 0.51 & -31.43 & 0.88 & 3.97 \\
\hline SMS-5 & OM-rich Mn carbonate & 1479.3 & -10.98 & 0.55 & -8.35 & 0.77 & -31.59 & 0.03 & 3.2 \\
\hline SMS-11 & OM-rich Mn carbonate & 1477.3 & -8.15 & 0.15 & -9.01 & 0.11 & -31.51 & 0.17 & 3.33 \\
\hline SMS-19 & OM-rich Mn carbonate & 1475.1 & -6.7 & 0.12 & -9.17 & 0.08 & -31.49 & 0.01 & 2.95 \\
\hline
\end{tabular}

Table 3. Cd isotope compositions and $\mathrm{Cd}$ concentrations derived from isotope dilution (ID).

\begin{tabular}{|c|c|c|c|c|c|c|c|c|}
\hline Sample ID & Depth (m) & Lithology & Leach Sample & Reagent & Leached Phase & $\varepsilon^{112 / 110} \mathrm{Cd}_{\mathrm{NIST}}$ & 2SE & Cd ID (ng) \\
\hline SMS-1 & 1481.3 & black shale & L1.1 & $1 \mathrm{MHAc}$ & carbonate & 0.66 & 1.1 & 235 \\
\hline SMS-3 & 1480 & OM-rich Mn carbonate & L1.3 & $1 \mathrm{M} \mathrm{HAc}$ & carbonate & -1.0 & 2.9 & 53.2 \\
\hline SMS-5 & 1479.3 & OM-rich Mn carbonate & L1.5 & $1 \mathrm{M} \mathrm{HAc}$ & carbonate & -0.48 & 0.5 & 280 \\
\hline SMS-11 & 1477.3 & OM-rich Mn carbonate & L1.11 & $1 \mathrm{M} \mathrm{HAc}$ & carbonate & 0.14 & 0.3 & 48.9 \\
\hline SMS-19 & 1475.1 & OM-rich Mn carbonate & L1.19 & $1 \mathrm{M} \mathrm{HAc}$ & carbonate & 0.48 & 0.2 & 49.9 \\
\hline SMS-1 & 1481.3 & black shale & $\mathrm{L} 2.1$ & $2 \mathrm{M} \mathrm{HCl}$ & $\mathrm{OM}$ & 2.9 & 1.4 & 64.0 \\
\hline SMS-3 & 1480 & OM-rich Mn carbonate & L2.3 & $2 \mathrm{M} \mathrm{HCl}$ & $\mathrm{OM}$ & 0.67 & 0.6 & 10.6 \\
\hline SMS-5 & 1479.3 & OM-rich Mn carbonate & $\mathrm{L} 2.5$ & $2 \mathrm{M} \mathrm{HCl}$ & $\mathrm{OM}$ & 0.13 & 0.9 & 178 \\
\hline SMS-11 & 1477.3 & OM-rich Mn carbonate & L2.11 & $2 \mathrm{M} \mathrm{HCl}$ & $\mathrm{OM}$ & 1.5 & 0.2 & 12.8 \\
\hline SMS-1 & 1481.3 & black shale & L3.1 & $1 \mathrm{M} \mathrm{NaOH}$ & sulphate & n.a. & n.a. & \\
\hline SMS-3 & 1480 & OM-rich Mn carbonate & L3.3 & $1 \mathrm{M} \mathrm{NaOH}$ & sulphate & n.a. & n.a. & \\
\hline SMS-5 & 1479.3 & OM-rich Mn carbonate & L3.5 & $1 \mathrm{M} \mathrm{NaOH}$ & sulphate & n.a. & n.a. & \\
\hline SMS-11 & 1477.3 & OM-rich Mn carbonate & L3.11 & $1 \mathrm{M} \mathrm{NaOH}$ & sulphate & n.a. & n.a. & \\
\hline SMS-19 & 1475.1 & OM-rich Mn carbonate & L3.19 & $1 \mathrm{M} \mathrm{NaOH}$ & sulphate & n.a. & n.a. & \\
\hline SMS-1 & 1481.3 & black shale & L4.1 & Aqua Regia & sulphide & 1.5 & 0.4 & 166 \\
\hline SMS-3 & 1480 & OM-rich Mn carbonate & L4.3 & Aqua Regia & sulphide & 0.92 & 0.8 & 154 \\
\hline SMS-5 & 1479.3 & OM-rich Mn carbonate & L4.5 & Aqua Regia & sulphide & 3.2 & 1.7 & 155 \\
\hline SMS-11 & 1477.3 & OM-rich Mn carbonate & L4.11 & Aqua Regia & sulphide & 0.8 & 0.3 & 156 \\
\hline SMS-19 & 1475.1 & OM-rich Mn carbonate & L4.19 & Aqua Regia & sulphide & 1.0 & 0.1 & 237 \\
\hline SMS-3 & 1480 & OM-rich Mn carbonate & L5.3 & conc. $\mathrm{HF}+\mathrm{HNO} 4$ & silicate & -0.34 & 1 & 15.5 \\
\hline SMS-5 & 1479.3 & OM-rich Mn carbonate & L5.5 & conc. $\mathrm{HF}+\mathrm{HNO} 5$ & silicate & n.a. & n.a. & n.a. \\
\hline SMS-11 & 1477.3 & OM-rich Mn carbonate & L5.11 & conc. $\mathrm{HF}+\mathrm{HNO} 6$ & silicate & n.a. & n.a. & n.a. \\
\hline SMS-19 & 1475.1 & OM-rich Mn carbonate & L5.19 & conc. $\mathrm{HF}+\mathrm{HNO} 7$ & silicate & n.a. & n.a. & n.a. \\
\hline
\end{tabular}




\section{Discussion}

\subsection{The Leaching of Mixed Marine Lithologies: Bioavailable Trace Metal Distributions Associated with (In)} Organic Phases

This section highlights and investigates the distribution of bioavailable trace metals $\mathrm{Ba}, \mathrm{Mo}$, $\mathrm{Cd}, \mathrm{Zn}, \mathrm{Co}, \mathrm{Mn}, \mathrm{Mo}, \mathrm{Ni}$, and $\mathrm{Cu}$ as well as $\mathrm{Zr}$ and $\mathrm{Ti}$ that are almost immobile elements clearly associated with detrital aluminosilicates between the five operational leaching phases L1 to L5. We postulate that the leaching step 1 attacked abundant Mn carbonate minerals such as rhodochrosite and calico-rhodochrosite that have been reported for the mixed lithologies of the basal Datangpo Fm. at Daotuo [24,26] and resulted in high absolute Mn concentrations obtained in L1. Further, elevated average $\mathrm{Ni}, \mathrm{Cd}, \mathrm{Co}$, and $\mathrm{Cu}$ concentrations in the leachate (making up $31 \%, 30 \%, 20 \%$, and $15 \%$ of the total budget, respectively) may be explained by the substitution of these metals for $\mathrm{Ca}^{2+}$ into carbonate lattices [63,76,77]. In L1, concentrations of Ti and Zr were exceptionally low and, in the case of $\mathrm{Zr}$, below the detection limit, arguing against leaching of any detrital material. L2 was supposed to leach remaining carbonates as well as the labile metal compounds bound onto the fulvic acid compounds of OM that were extractable by strong acids. The metal concentrations and distributions obtained were usually in the range of L1 with the exceptions of Mo (up to $9 \mu \mathrm{g} / \mathrm{g}$ and 20\%) which can be attributed to Mo affinity to organic complexes. L3 described leaching of sulphate components and showed low trace metal concentration levels (below $1 \mu \mathrm{g} / \mathrm{g}$ ). Only $\mathrm{Zn}$ and Ba were enriched and made up 34\% and 55\% of the bulk budget, respectively. L4 broke down sulphides, which were abundant as framboidal authigenic pyrite grains in the shales and of the lower Datangpo Fm. (see EBSD pictures in Supplementary Materials Figures 1 and 2 and the existing literature on the lower Datangpo Fm. $[11,22,24])$. The metal concentrations in L4 were high; however, distributions varied distinctively between the bioavailable metals: While concentrations of up to $0.27 \mu \mathrm{g} / \mathrm{g}$ made up $50 \%$ of the whole rock Cd budget, Zn concentrations of $19 \mu \mathrm{g} / \mathrm{g}$ only accounted for $12 \%$ of the whole rock concentration bound in sulphides. Further, concentrations of Mo, Ni, and $\mathrm{Cu}$ were high in L4 and accounted for the highest or second highest proportion of the bulk metal concentration. L5 fully digested the remaining detrital silicates reported for the basal Datangpo Fm. [24]. High Ti concentrations of 1114 to 17,049 $\mu \mathrm{g} / \mathrm{g}$ argued for the dissolution of the remaining silicate minerals. The obtained metal concentrations obtained in L5 were highly variable throughout the different sample set. High variations within the sample suite were observed in $\mathrm{Zn}, \mathrm{Cd}, \mathrm{Ni}$, and $\mathrm{Cu}$ where concentrations of leaching step 5 in sample SMS-11 exceeded all other samples by up to one order of magnitude.

It is notable that average $\mathrm{Cd}, \mathrm{Mo}, \mathrm{Zn}$, and Ba concentrations of the $\mathrm{L} 5$ matched the reported values for the upper continental crust (UCC) [78] while $\mathrm{Cu}, \mathrm{Zn}$, and Mn only overlapped in their total obtained range with UCC values. $\mathrm{Cu}$ and $\mathrm{Zn}$ revealed slightly lower concentrations in the silicate leach than anticipated for the average UCC composition. However, the differences were small and may reflect different hinterland compositions distributing variable detrital material into the Nanhua Basin or UCC values that were based on bulk rock analyses which comprised a suite of different minerals including nonsilica phases. We, therefore, argue that the L5 leachate represents the best representative for the local detrital background in the Nahua Basin at the beginning of the Datangpo Formation.

\subsection{The Redox State and (Bio)Geochemical Metal Cycling in the Cryogenian Yangtze Ocean}

All analysed trace metal concentrations in sequential leachates from the Cryogenian Datangpo OM-rich shale and Mn carbonates were normalized to the local detrital hinterland composition to diminish the impact of detrital material on the chemical sediment's signal. Hence, redox-sensitive element enrichments gave us information about (1) the relative enrichment of the element dissolved in the ambient bottom/pore water represented by the carbonate leachate, (2) the relative enrichment in undissolved OM and sulphates, and (3) the relative enrichment of the element leached authigenic and/or diagenetic sulphides. Our results showed that relative depletion of $\mathrm{Ba}, \mathrm{Zn}$, and Mo together with a more than 10-fold relative enrichment of Mn into carbonate phases argues for a reducing depositional 
environment in which Mn-oxides dissolved, releasing excess Mn into the ambient seawater. Together with prominent enrichments in Mn in the carbonate leach but coincident depletions in the sulphide leachate of $\mathrm{Mn}$ this argues for anoxic and manganous conditions in the bottom/pore waters at the deposition of the studied samples. The leached $\mathrm{OM}$ was generally enriched in the vital trace metals $\mathrm{Cu}$, $\mathrm{Mo}, \mathrm{Mn}$, and $\mathrm{Cd}$, arguing for adsorption onto $\mathrm{OM}$ or nutrient-like uptake in phototrophic organisms. By contrast, sulphate leachate L3, i.e., sedimentary barites and (oxy)thiomolybdates, showed depletion in $\mathrm{Cu}, \mathrm{Co}, \mathrm{Mn}$, and $\mathrm{Cd}$ but enrichment in $\mathrm{Zn}, \mathrm{Ba}$, and $\mathrm{Mo}$ due to their deposition under slightly euxinic conditions in the pore water space [41]. Mo had low affinity to be incorporated into $\mathrm{CaCO}_{3}$, while it can be adsorbed onto positively charged organic particles and incorporated into (oxy)thiomolybdates or sulphides [41]. Consequently, our carbonate leachate L1 showed low Mo concentrations while the sulphide leachate L3 showed higher Mo concentrations, further arguing for euxinic pore water/bottom water conditions. This finding is further supported by the high $\delta^{98}$ Mo values recorded at the base of the Datangpo Fm. [21]. The enrichments of $\mathrm{Cd}, \mathrm{Cu}, \mathrm{Ni}$, and $\mathrm{Co}$ in the sulphide leach argue for incorporation under euxinic conditions being present in the ambient fluids, supported by the presence of framboidal sulphide grains (EBSD pictures in Supplementary Materials Figure S2).

Iron speciation and S isotope analyses [10] suggest that the early Cryogenian Nanhua Basin can be best explained by a vertically stratified redox model. However, variable $U$ and Mo enrichments in lower Datangpo Fm. shales in combination with shale-normalized REY patterns showing distinct positive $\mathrm{Ce}$ and negative $\mathrm{Y}$ anomalies argue for at least episodic oxic to suboxic conditions in the basin that allowed for the formation of Mn oxides [22,24] as well as Fe oxides [11] somewhere in the water column. While iron speciation and S isotope analyses [10] showed that the early Cryogenian Nanhua Basin can be best explained by a vertically stratified redox model, variable $U$ and Mo enrichments in lower Datangpo Fm. shales as well as shale normalized REY patterns having positive Ce and negative $\mathrm{Y}$ anomalies argued for at least episodic oxic to suboxic episodes in the basin that allowed for the formation of Mn oxides [22,24] as well as Fe oxides [11] somewhere in the water column.

The element distribution in Cryogenian seawater in the Nanhua Basin can be represented by leachates of OM-rich shale and Mn carbonates building up the basal Datangpo Fm. which most likely precipitated under manganous to slightly euxinic bottom water conditions and under the presence of a massive DOC pool [11,20-23]. Under reducing conditions many vital trace elements (TE), although potentially abundant in Nanhua Basin seawater (given the overlap in elemental concentration of L5 with UCC), were adsorbed onto sinking OM or bound in sedimentary sulphides or thiomolybdates $(\mathrm{Zn}, \mathrm{Ba}, \mathrm{Mo}$ ) and not recycled in the water column. However, relative enrichments in $\mathrm{Co}, \mathrm{Cu}, \mathrm{Ni}, \mathrm{Cd}$, and $\mathrm{Mn}$ in the carbonate leachates reveal that some critical trace metals may have had the chance to be recycled and to remain in the water column and have been available for marine microbial life during formation of the basal Datangpo Fm.

\subsection{The Fractionation of Stable Cd Isotopes in Authigenic Phases: Implications on Contemporaneous Cryogenian Nanhua Basin Surface and Deep Waters}

\subsubsection{The Reliability of Cd Isotopes in Leachates from Mixed Lithology Samples}

The use of authigenic mineral phases as archives for ancient environmental conditions strongly relies on the assumption that the pristine elemental budget of the mineral of interest is not masked by detrital contamination and/or post-depositional alterations during fluid-rock interactions. Cadmium isotopic compositions vs. Cd concentrations (Figure 4) and O isotopic compositions (Supplementary Materials Figure S5) in the leached phases showed neither correlations with highly immobile elements (e.g., $\mathrm{Al}, \mathrm{Zr}$, Ti) that were clearly associated with detrital material nor with fluid mobile elements (e.g., $\mathrm{Sr}, \mathrm{O}) . \mathrm{Cd} / \mathrm{Ti}$ ratios found in nondetrital phases were three orders of magnitude higher than values reported in loess as a representative for the UCC composition (Figure $4 \mathrm{a}, \mathrm{f})$. Cd isotopic composition of the HF leaching step further overlapped with the upper continental crust (Figure 4f), indicating that the results of this leaching step most closely represented the local detrital input from the continental hinterland into the Nanhua Basin. However, both $\mathrm{Cd} / \mathrm{Zn}$ ratios and $\mathrm{Cd}$ isotope compositions of 
carbonate, $\mathrm{OM}$, and sulphide leachates substantially differed from the siliciclastic leachates and average UCC values, suggesting that detrital material had negligible impact on the elemental and isotopic budget of the carbonates. Furthermore, the lack of positive correlations between $\mathrm{Cd}$ concentrations and the isotopic compositions of all leached mineral phases with highly fluid mobile $\mathrm{Sr}$ and $\mathrm{O}$ isotopes (Supplementary Materials Figure S5) strongly suggest that post-depositional alteration during diagenesis or metamorphic processes did not affect the $\mathrm{Cd}$ budget of the here-studied mineral phases. Thus, carbonate fractions reliably traced the $\mathrm{Cd}$ budget from the fluid of which they were precipitated and can be used as prime geochemical archives to reconstruct (bio)geochemical metal cycling processes within the Nanhua Basin.
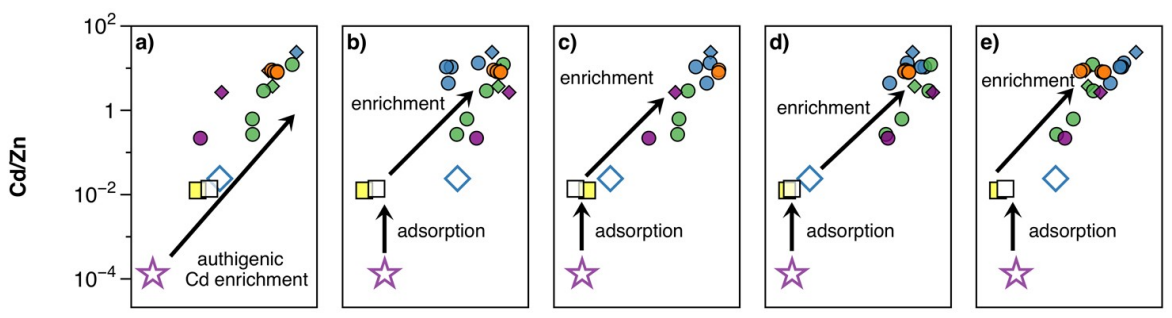

- $1 \mathrm{MHAC}$

- $2 \mathrm{M} \mathrm{HCl}$

conc. Aq. Reg.

o conc. $\mathrm{HF}+\mathrm{HNO}_{3}$

$\diamond$ CAL-S; Phan. carb. RM

ts UCC

$\checkmark$ NOD-A-1; USGS RM

$\square$ NOD-P-1; USGS RM

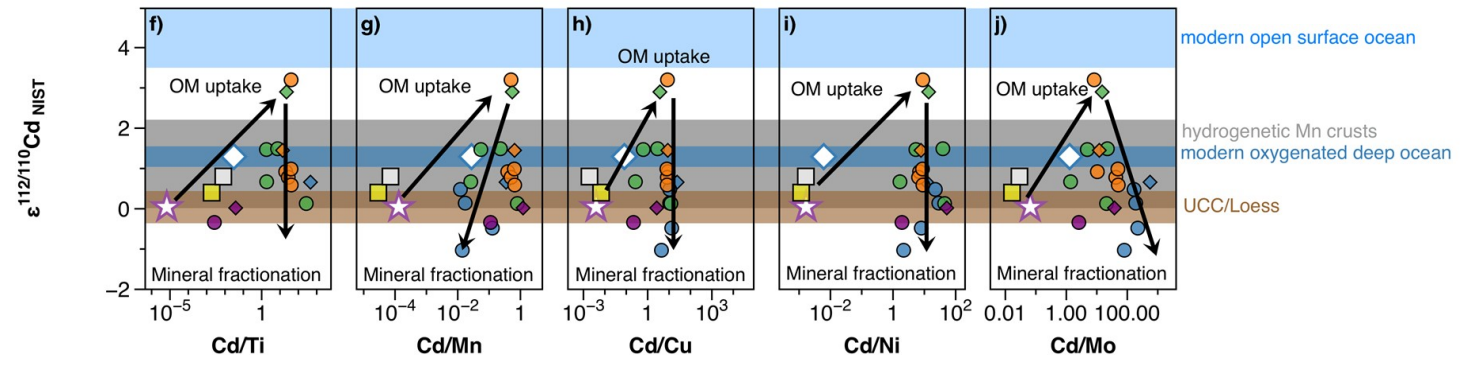

Figure 4. (a-e) $\mathrm{Cd} / \mathrm{Zn}$ ratios relative to $\mathrm{Cd}$ over $\mathrm{Ti}, \mathrm{Cu}, \mathrm{Ni}, \mathrm{Mn}$, and $\mathrm{Mo}$ of leachate steps $\mathrm{L} 1$ to $\mathrm{L} 5$. $(\mathbf{f}-\mathbf{j})$ stable $\mathrm{Cd}$ isotope compositions relative to $\mathrm{Cd}$ over $\mathrm{Ti}, \mathrm{Cu}, \mathrm{Ni}, \mathrm{Mn}$, and $\mathrm{Mo}$ of leachate steps L1 to L5. Diamonds represent the respective compositions obtained in the analysed shale lithology. Loess data [56], surface ocean [79-81], deep ocean [54], and hydro genetic Mn crusts [82]. Long-term reproducibility of spiked Cd isotope reference material NIST SRM 3108 is $0.4 \varepsilon$ units (2SD).

\subsection{2. (Bio)Geochemical Cycling of Cd Isotopes in the Nanhua Basin}

Cross-plots of $\mathrm{Cd}$ isotope compositions vs. Cd concentrations over trace element concentration plots (Figure 4) show a systematic behaviour of isotope fractionation and element incorporation between the studied leachates and comparable marine and crustal rock standard reference materials. We here discuss that three general processes, i.e., (1) authigenic $\mathrm{Cd}$ enrichment trend, (2) $\mathrm{Cd}$ adsorption trend, and (3) biologic $\mathrm{Cd}$ enrichment trend, control incorporation, and fractionation of $\mathrm{Cd}$ and its isotopes in paleo-marine mixed sediment records. In Figure $4 \mathrm{a}$, a linear authigenic $\mathrm{Cd}$ enrichment trend (I) can be seen between $\mathrm{Cd} / \mathrm{Zn}$ ratios and $\log [\mathrm{Cd} / \mathrm{Ti}]$ ratios. The trend steadily increases from a loess composition analysed by Schmitt et al. (2009) [56] that we used as the best representative for a upper continental crust (UCC) via marine ferro-manganese nodules [82] and the Phanerozoic marine carbonate reference material CAL-S [30] towards our values obtained in individual leachates L1 to L5 from the basal Datangpo formation. We argue that this authigenic Cd enrichment trend (1) was mainly governed by the incorporation of $\mathrm{Cd}$ into $\mathrm{OM}$ in the water column and accumulation in authigenic phases in marine sediments [52] relative to the UCC and Mn nodules (hence authigenic nondetrital phases showed lower Ti concentrations). Two further trends can be observed: The Cd adsorption trend (2) is defined by a straight increase of $\mathrm{Cd} / \mathrm{Zn}$ ratios with no relative variation in $\mathrm{Cd}$ over $\mathrm{Mn}, \mathrm{Cu}, \mathrm{Ni}$, and Mo rations, respectively (Figure $4 \mathrm{~b}-\mathrm{e}$ ). Even though $\mathrm{Zn}$ tended to be adsorbed onto ferro-manganese crusts under oxic conditions, its adsorption and enrichment factor relative to ambient seawater was less than those of the other bioavailable trace metals [83]. The positive-correlated biologic $\mathrm{Cd}$ enrichment trend (3) between $\mathrm{Cd}$ and other bioavailable trace metals, which can be seen 
for authigenic formed marine minerals such as sulphides and carbonates as well as for OM, was most likely controlled via $\mathrm{Cd}$ uptake. Further, adsorption of $\mathrm{Cd}$ onto clay minerals such as illite has been described [84], that may denote for the L5 leachate element ratios plotting on the Cd enrichment trend as well. $\mathrm{Cd}$ isotope compositions help us to further understand the processes of $\mathrm{Cd}$ enrichment in the leachates from OM-rich mixed marine sediments. Figure $4 \mathrm{f}-\mathrm{j}$ show similar trends between $\mathrm{Cd}$ isotope compositions and $\mathrm{Cd}$ over trace metal ratios. In Figure $4 \mathrm{f}$ a positive correlation between $\varepsilon^{112 / 110} \mathrm{Cd}$ vs. $\mathrm{Cd} / \mathrm{Ti}$ indicates that an isotopic fractionation took place during the uptake of $\mathrm{Cd}$ into $\mathrm{OM}$ that further enriched the $\mathrm{Cd}$ relative to $\mathrm{Ti}$ in the biomass. The surface water in which phototrophic organism utilized the $\mathrm{Cd}$ as a result was extremely depleted in $\mathrm{Cd}$ and had a heavy $\mathrm{Cd}$ isotope composition of up to 4 to $6 \varepsilon^{112 / 110} \mathrm{Cd}$ values (light blue shade in Figure $4 \mathrm{f}-\mathrm{j}$ ) $\mathrm{cf}$. $[54,79,85,86]$. The oxidative decay of sinking dead biomass led to a replenishment of $\mathrm{Cd}$ in deep water and further $\mathrm{Cd}$ fractionation into marine authigenic minerals such as $(\mathrm{Mn})$ carbonates and sulphides precipitated under reducing and euxinic conditions, respectively $[45,46,85,87]$. The secondary formed minerals tended to have the same $\mathrm{Cd} / \mathrm{Ti}$ ratios, however, they showed distinct lighter $\mathrm{Cd}$ isotope compositions than the (deep) ocean waters they formed in. This argues for second order fractionation of light isotopes into authigenic minerals that has been described for carbonates [64] and sulphides [45], respectively. While Figure 4 h,i show the same trend of $\varepsilon^{112 / 110} \mathrm{Cd}$ vs. stagnant $\mathrm{Cd} / \mathrm{Cu}$ and $\mathrm{Cd} / \mathrm{Ni}$ ratios (arguing for nearly identical incorporation behaviour of the metals into authigenic minerals), Figure $4 \mathrm{~g}$ shows a negative correlation of $\varepsilon^{112 / 110} \mathrm{Cd}$ with $\mathrm{Cd} / \mathrm{Mn}$, which can be attributed to the formation of Mn carbonates under manganous deep/pore water conditions in the Nanhua Basin during the deposition of the basal Datangpo Fm. Finally, Figure 4j shows the opposite trend with increasing Cd/Mo vs. decreasing $\varepsilon^{112 / 110} \mathrm{Cd}$, which may be attributed to the low affinity of Mo to be incorporated into carbonate lattice.

\subsubsection{Assessing the Cd Isotope Composition of Cryogenian Nanhua Basin Surface and Deep Seawater}

The Cryogenian Yangtze Ocean was situated at the banks of the Yangtze Craton which had a passive continental margin with a SW facing slope towards the open ocean. On this continental shelf environment, several protracted basins developed in the terminal Proterozoic that were periodically restricted from the open ocean via NE to SW striking tectonic barriers. While Cryogenian oceans already witnessed the rise of phototrophic algae [17], the temporally restricted exchange of waters in the Nanhua Basin led to stratified redox conditions with oxic to suboxic surface waters overlying anoxic to euxinic deep waters $[10,25]$. Under these circumstances, a large organic carbon pool built up in the deep ocean which was only periodically and partially oxidized [25], limiting large amounts of OM-associated bio-essential trace metals for nutrient recycling. Examples for modern analogues to such an environment of high phototrophic primary productivity with redox stratification are the Black Sea, some Norwegian fjords, the Peruvian Margin, and the Cariaco Trench. Although scarce, few existing $\mathrm{Cd}$ isotope datasets in these environments hint to combined effects of the above described biologic $\mathrm{Cd}$ uptake, causing Cd depleted 'heavy' $\varepsilon^{112 / 110} \mathrm{Cd}_{\mathrm{SSW}}$ surface seawaters (SSW) and Cd enriched 'light' $\varepsilon^{112 / 110} \mathrm{Cd}_{\mathrm{DW}}$ deep waters (DW) due to $\mathrm{Cd}$ release via sinking OM decay. The Cd uptake in modern closed and semi-closed marine systems such as the Southern Ocean and the Black Sea is described to follow simple Rayleigh-distillation models with fractionation factors $\alpha^{112 / 110} C d_{S S W-O M}$ between 1.0001 to 1.0006 [79,85,88]. Further, laboratory experiments with Cd stock solutions and freshwater phytoplankton Chlamydomonas reinhardtii and Chlorella spirulina by Lacan et al. (2006) [55] supported fractionation factors in the same order. Figure 5a shows a simplified model for modern oxygenated oceans with constant fractionation of a the $\mathrm{Cd}$ pool in the surface waters under semi-closed to closed system Rayleigh-type biologic fractionation (i.e., with or without Cd replenishment) [88]. Here an averaged fractionation factor of $\alpha_{S S W-O M}=1.00035$ was used to illustrate fractionation of Cd into biomass via biological uptake [79]. An additional fractionation factor of $\alpha_{S S W-c a r b}$ of 1.000227 [64] for the kinetic uptake of the remaining $\mathrm{Cd}$ in the surface waters into biogenic carbonates, i.e., foraminifera tests, was assumed. For example, in the Cd supply-limited modern Southern Ocean's surface water the $\mathrm{Cd}$ isotope compositions were in the order of +4 to $+6 \varepsilon^{112 / 110} \mathrm{Cd}$ units, while deep water compositions 
ranged between +1 and $+2 \varepsilon^{112 / 110} \mathrm{Cd}$ [79]. Together, Cd-depleted surface waters and Cd-enriched deep waters mimic the typical seawater macro-nutrient phosphorous' depth concentration gradient (purple line Figure 5a) [89]. These patterns can be best explained by oxidative $\mathrm{Cd}$ release from sinking degrading $\mathrm{OM}$, further leading to the recycling of $\mathrm{Cd}$ and potentially other bio-essential trace metals [80]. However, negative deviations of the analysed $\mathrm{Cd}$ content in deep waters from the expected concentrations have been linked to oxygen depletion zones (ODZ) $\left(<100 \mu \mathrm{mol} / \mathrm{L} \mathrm{O}_{2}\right)$ and the presence of free $\mathrm{H}_{2} \mathrm{~S}$ [46]. Under sulphidic conditions, additional $\mathrm{Cd}$ isotope fractionation into $\mathrm{Cd}$ sulphides has been described in a $\mathrm{H}_{2} \mathrm{~S}$ plume near the Peruvian Margin [85] as well as in the euxinic deep waters of the Black Sea [87]. Thus, these individual and combined fractionation factors have the potential to shift the typical "nutrient-like" $\varepsilon^{112 / 110} \mathrm{Cd}$ depth gradient to heavier values in modern oxygen-depleted deep water environments (Figure 5a, dashed purple line).
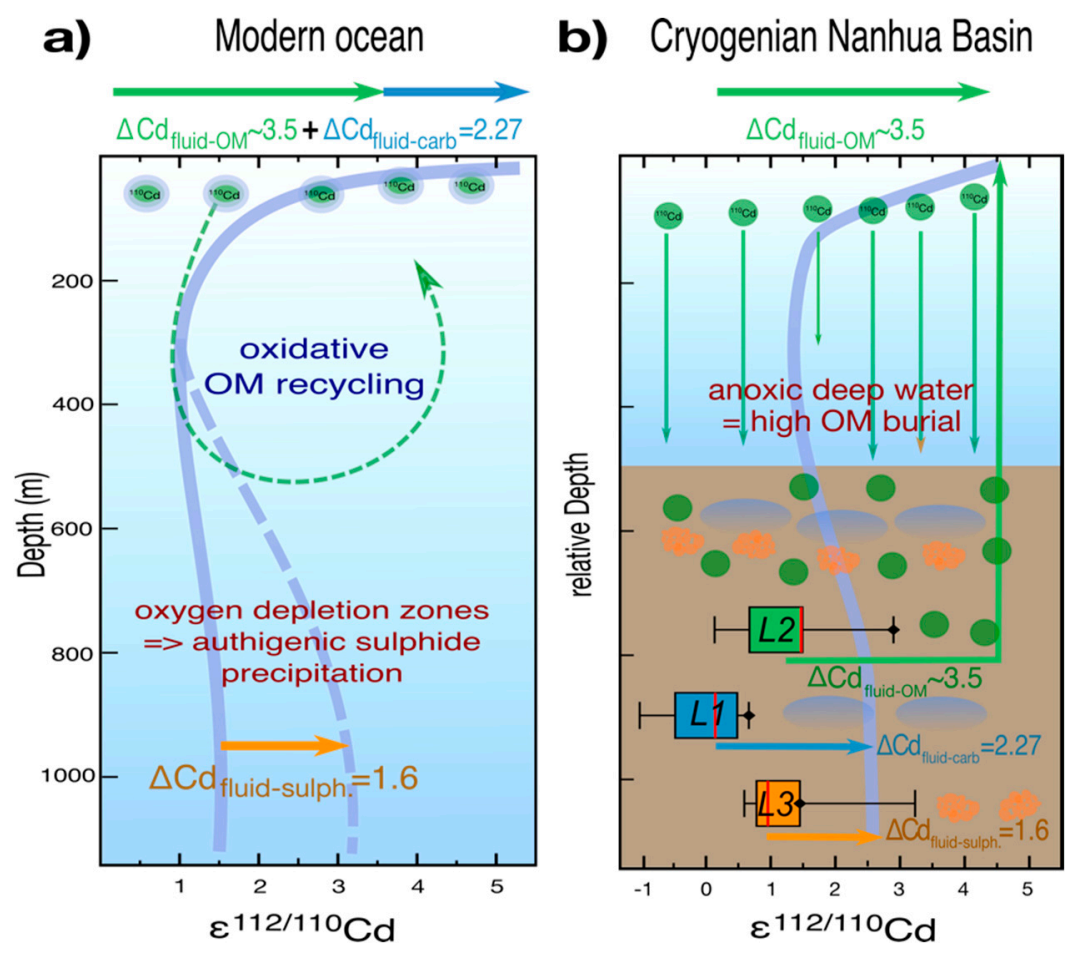

Figure 5. Behaviour of Cd isotopes in the modern ocean (a) and in the Cryogenian Nanhua Basin (b). (a) Schematic model for the Cd isotope 'nutrient-like' depth profile in the modern oceans. Cd isotopes are fractionated from the continental derived input in the photic zone via biological uptake leading to progressively 'heavy' Cd-enriched surface waters. This effect may be accompanied by fractionation of the $\mathrm{Cd}$ pool into carbonate tests with a further positive fractionation factor. Sinking dead biomass within the water column can be oxidized and the $\mathrm{Cd}$ gets recycled, causing modern deep waters to have 'lighter' Cd isotope compositions. In oxygen minimum zones, the formation of authigenic Cd sulphides under the presence of free $\mathrm{H}_{2} \mathrm{~S}$ can cause further isotope fractionation and a shift to 'heavier' deep water values. (b) Schematic model of a theoretical Cd isotope profile in the redox stratified Cryogenian Nanhua Basin. Only Cd utilization by algae may fractionate the surface waters' Cd pool in the photic zone while carbonate shells were not existent. In the anoxic deep and pore waters of the Nanhua Basin the formation of authigenic carbonates and sulphides (represented by the obtained values in the L1 and L3 leachates) shifted deep water Cd isotope compositions that were in isotopic equilibrium with the DOC (recorded in the obtained values from OM leachate L2) to 'heavier' values that are above the values recorded in modern oxygenated deep oceans.

In order to model the surface seawater for the stratified Cryogenian ocean at the time of the basal Datangpo Fm. deposition we assumed that the OM leachate L2 recorded the Cd isotope composition of the phototrophic biomass that fractionated the Cd pool in the Nanhua Basin photic zone. Perhaps the 
simplest approach would be to use the average value of the obtained $\varepsilon^{112 / 110} \mathrm{Cd}$ values from $\mathrm{L} 2$ and add a constant average isotopic offset $\Delta^{112 / 110} C d_{S S W-O M}=+3.5$ derived from average $\alpha^{112 / 110} C d_{S S W-O M}$ obtained in modern surface ocean water. This calculation would place the $\varepsilon^{112 / 110} \mathrm{Cd}_{\mathrm{SSW}}$ of the Nanhua Basin at $\sim+4.5$ which is in the order of the modern oxygenated surface ocean $[54,79,81,85]$. Figure $5 \mathrm{~b}$ shows a whisker plot of the $\mathrm{Cd}$ isotope compositions obtained in three different operational leaching phases L1, L2, and L4 representing carbonate, OM, and sulphide leachates. Following [90], authigenic or early diagenetic (Mn) carbonates and sulphides in mixed OM-rich lithologies have the potential to record deep water/pore water compositions that are in equilibrium with dissolved organic carbon (DOC). From this carbon pool, and under the reductive dissolution of Mn oxy-hydroxides, inorganic carbonates such as $\mathrm{Mn}$ and Fe carbonates can precipitate under manganous/ferruginous conditions and, in the presence of free $\mathrm{H}_{2} \mathrm{~S}$, authigenic sulphides form within the sediment under euxinic conditions. EBSD pictures from the studied lithologies presume that thin horizons of Mn carbonates have indeed been formed in early diagenetic stage from colloidal Mn oxy-hydroxides (Supplementary Materials Figure S3). Further, the presence of Mn oxy-hydroxides as precursors for the studied Mn carbonates is underlined by rare earth element studies by [24] showing that Cryogenian Mn ores have similar REY patters but slightly enriched total REY concentrations comparable to modern ferromanganese crusts and nodules. The abundant framboidal sulphides (Supplementary Materials Figures S1 and S2) are closely associated to the Mn carbonate horizons arguing for their formation under progressively reducing/sulfidic conditions in the sediment. If the degrading OM was in isotopic equilibrium with the pore waters, instantaneous formed carbonates and sulphides would then be represented by an offset of $\Delta \mathrm{Cd}_{\text {fluid-carb }}=2.27$ and $\Delta \mathrm{Cd}_{\text {fluid-CdS }}=1.6$, respectively. This gave us the chance to estimate the Cryogenian $\varepsilon^{112 / 110} \mathrm{Cd}$ DW of the Nanhua Basin at the time of deposition which was in the order of $\varepsilon^{112 / 110} \mathrm{Cd}_{\mathrm{dw}}=\sim 2.5$. This value was significantly heavier than those values reported for modern oxygenated open ocean $[79,81]$ although only few $\mathrm{Cd}$ isotope datasets from redox stratified modern oceanic environments exist. We here, therefore, present a modelling approach for calculation of theoretic surface seawater as well as deep water values for the Cryogenian Nanhua Basin that can be easily adapted to modern redox stratified marine environments using simple mass balance equations following John et al. (2019) [58] using the following two assumptions as prerequisite: (1) There is one dominant source of $\mathrm{Cd}$ into the oceans which is continental weathering leading to a homogenous input into the oceans of $\varepsilon^{112 / 110} \mathrm{Cd}_{\mathrm{UCC}}=\sim 0$ [56] via riverine runoff and aeolian dust [91], and (2) three main $\mathrm{Cd}$ sinks exist in the Nanhua Basin which are $\mathrm{OM}$, as well as authigenic and early diagenetic $(\mathrm{Mn})$ carbonates and cadmium sulphides (CdS). It has been previously shown that the overall fractionation of $\mathrm{Cd}$ into a calcite/dolomite lattice under saline open marine conditions is in the order of $\Delta^{112 / 110} \mathrm{Cd}_{\text {fluid-carbonate }}=-2.27$ [64] and insensitive to the cooperation of other major cations like $\mathrm{Mg}^{2+}$. Guinoiseau et al. (2018) [45] showed that Cd isotope fractionation into CdS under saline fully marine conditions is $\Delta^{112 / 110} \mathrm{Cd}_{\text {fluid-CdS }}=-1.6$. Finally, the fractionation of $\mathrm{Cd}$ from the surface seawaters into phototrophic biomass is unknown and has to be assessed: The heaviest observed $\mathrm{Cd}$ isotope value in the Datangpo Fm. was about $+2.9 \mathrm{Cd} \varepsilon^{112 / 110} \mathrm{Cd}$ obtained in $\mathrm{L} 2$, arguing for an overall fractionation of $\mathrm{Cd}$ isotopes in the same order into $\mathrm{OM}$. This value is only slightly smaller than the observed $\mathrm{Cd}$ fractionation in modern surface oceans (presumably governed by nonquantitative uptake into phototrophic organisms) which is in the order of 3.5 to $6.5 \mathrm{Cd} \varepsilon^{112 / 110} \mathrm{Cd}$ [79]. This information leads to the calculation of the $\mathrm{Cd}$ isotope composition of Cryogenian surface seawater compositions at time of deposition using Equation (3):

$$
\varepsilon^{112 / 110} \mathrm{Cd}_{\mathrm{SSW}}=\varepsilon_{112 / 110} \mathrm{Cd}_{\mathrm{UCC}}-f_{\mathrm{OM}} \times \Delta^{112 / 110} \mathrm{Cd}_{\mathrm{SSW}-\mathrm{OM}}
$$

where $f_{\mathrm{OM}}$ is anywhere between 0.01 and 1 . In the next step, the Nanhua Basin deep water composition will be modelled by the assumption that authigenic Mn carbonates and Cd sulphides precipitated under reducing deep marine conditions governed by the decomposition of OM leading to $\mathrm{Cd}$ release and subsequent 'lighter' $\mathrm{Cd}$ isotopic compositions of the DOC that is in isotopic equilibrium with the OM. Authigenic Cd sulphides and Mn carbonates tap this 'light' DOC Cd pool with a preferential 
kinetic incorporation of light isotopes into their mineral lattice. As a result, reducing deep waters show 'heavier' $\mathrm{Cd}$ isotope values relative to ambient surface waters. Equation (4) describes the $\varepsilon^{112 / 110} \mathrm{Cd}$ of the Nanhua Basins' deep waters:

$$
\varepsilon^{112 / 110} \mathrm{Cd}_{\mathrm{DW}}=\varepsilon^{112 / 110} \mathrm{Cd} \mathrm{OM}_{\mathrm{OM}}-\Delta^{112 / 110} \mathrm{Cd}_{\text {fluid-CdS }} \times f_{\mathrm{CdS}}-\Delta^{112 / 110} \mathrm{Cd}_{\text {fluid-carbonate }} \times f_{\text {carbonate }}
$$

where $f_{\mathrm{CdS}}+f_{\text {carbonate }}=1$. Given this equation, $\varepsilon^{112 / 110} \mathrm{Cd} \mathrm{SSW}_{\mathrm{S}}$ (at a given amount of Cd uptake by phototrophic biomass) with $\varepsilon^{112 / 110} \mathrm{Cd}$ DW under variable sulphide and carbonate formation in the deep water can be calculated. Figure 6A shows the evolution of the theoretical Nanhua Basin surface water vs. deep water $\mathrm{Cd}$ isotope compositions in coloured variable-sized circles. Increasing primary productivity shifts both the surface and the deep water $\varepsilon^{112 / 110} \mathrm{Cd}$ to heavier values (colour change from red to green in Figure 6A). While increased carbonate over CdS precipitation (big vs. small circles in Figure 6A) shifted both water masses, Cd compositions to even higher values due to the bigger fractionation factor were associated with $\mathrm{Cd}$ incorporation into the carbonate lattice. Green dashed and dash/dotted lines in Figure 6a represent possible evolution of the instantaneous formed biomass $\varepsilon^{112 / 110} \mathrm{Cd}_{\mathrm{OM}}$ values with $\alpha^{112 / 110} \mathrm{C} d_{S S W-O M}=1.0002$ [79] and 1.00035 [55]. We proposed earlier that deep waters of the Nanhua Basin can be considered to be in isotopic equilibrium with the DOC as long as no secondary authigenic mineral fractionation occurred that would have shifted the deep waters' composition to heavier values (orange and blue arrow in Figure 6A). Therefore, we applied two possible $\Delta^{112 / 110} \mathrm{Cd}$ DW-OM of +2 and +3.5 to calculate the compositions of surface waters without authigenic mineral formation. The results were plotted as green arrays of $1.5 \varepsilon$ units' length (difference between the two $\Delta^{112 / 110} \mathrm{Cd}$ DW-OM offsets) in Figure $6 \mathrm{~A}$, which overlap with potential OM surface water compositions derived from phototrophic primary production. Figure $6 \mathrm{~B}$ shows the Cd isotope composition vs. Cd concentration obtained in the L1 and L4 leachates of authigenic carbonate and sulphide, respectively (light shaded symbols), and the $\mathrm{Cd}$ composition of theoretic ambient fluid they precipitated off. For simplicity, theoretic $\mathrm{Cd}$ isotope compositions of the ambient deep/pore water from which authigenic or early diagenetic $\mathrm{Cd}$ sulphides and Mn carbonates instantaneously precipitated were calculated without concentration changes of the fluid during mineral precipitation:

$$
\begin{aligned}
& \varepsilon^{112 / 110} \mathrm{Cd}_{\text {fluid }}=\varepsilon^{112 / 110} \mathrm{Cd}_{\mathrm{CdS}}+\Delta \mathrm{Cd}_{\text {fluid-CdS }} \\
& \varepsilon^{112 / 110} \mathrm{Cd}_{\text {fluid }}=\varepsilon^{112 / 110} \mathrm{Cd}_{\text {carb }}+\Delta \mathrm{Cd}_{\text {fluid-carb }}
\end{aligned}
$$

Although the plotted ambient fluid $\varepsilon^{112 / 110} \mathrm{Cd}_{\text {fluid }}$ vs. $\log [\mathrm{Cd}]$ concentrations obtained in the respective authigenic minerals plot on a linear trend, i.e., are directly correlated, it is unlikely that this phenomenon represents a typical Rayleigh-type fractionation, because it is opposite to that observed in the modern oxygenated Southern Ocean [79] or ancient microbial carbonates [30]. In both cases, the heavy $\varepsilon^{112 / 110} \mathrm{Cd}_{\text {fluid }}$ correlated with high $\mathrm{Cd}$ concentrations arguing for closed-system Rayleigh-type fractionation via biomass $\mathrm{Cd}$ uptake leading to a $\mathrm{Cd}$ depletion in the ambient fluid. In the analysed authigenic Mn carbonate and sulphide leachates from the basal Datangpo Fm., however, heavier $\varepsilon^{112 / 110} \mathrm{Cd}_{\text {fluid }}$ values correlated with higher $\mathrm{Cd}$ concentrations, arguing for Cd incorporation into the mineral lattices with nonlimited Cd supply via OM decomposition. 


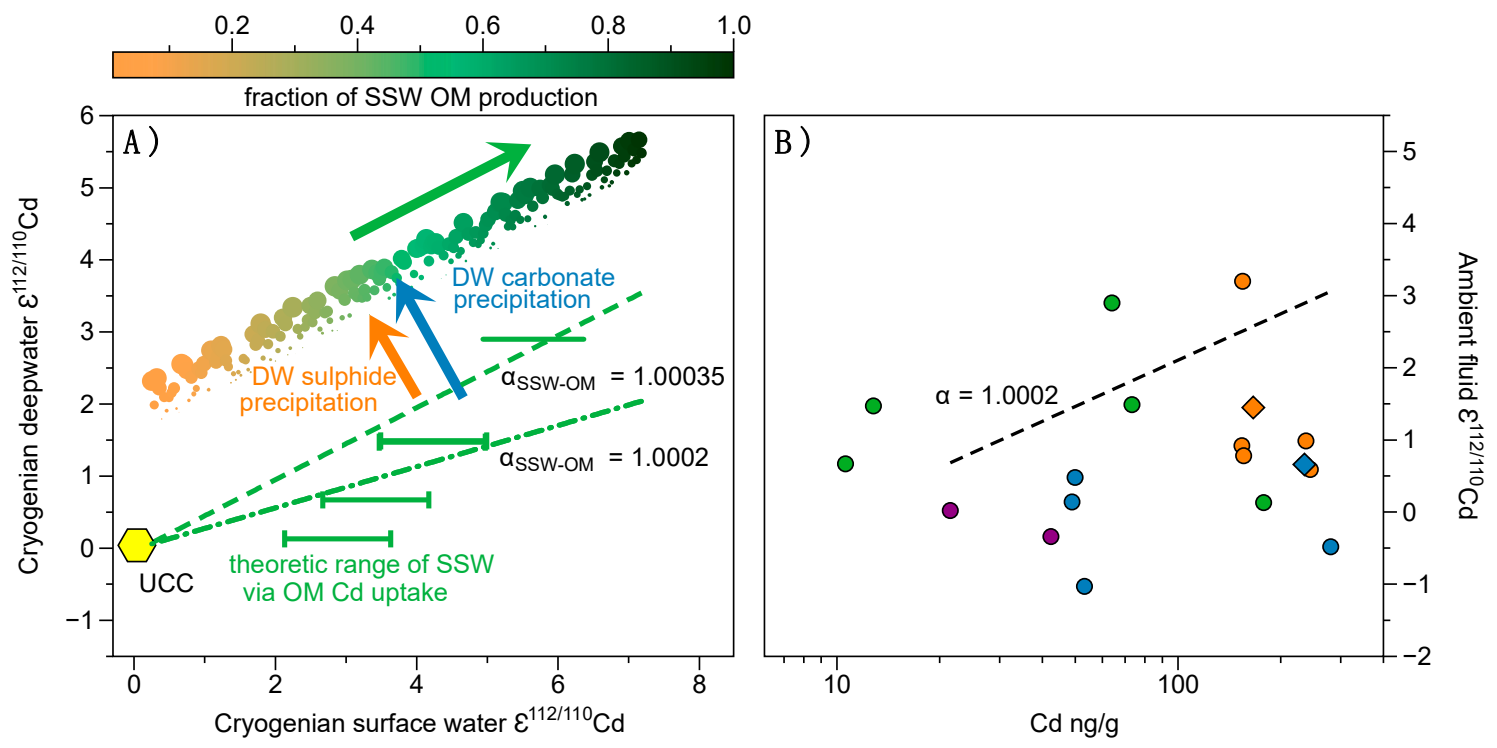

Figure 6. Modelled Cd isotope composition in the Cryogenian surface and deep water (A) with measured $\mathrm{Cd}$ concentrations relative to its $\mathrm{Cd}$ isotopic composition in individual mineral phases (B). (A) Modelled theoretical Cd isotopic compositions of surface seawater and deep water in a redox stratified restricted marine basin. The colour code from red to green represents increasing Cd uptake in the surface waters by phototrophic biomass. The size of the circles refers to the amount of carbonate over sulphide being precipitated out of deep water in isotopic equilibrium with a DOC pool. The instantaneously formed biomass $\mathrm{Cd}$ isotopic compositions are plotted as linear evolving trends (green dashed and dashed/dotted lines) from the initial Cd composition of the UCC [56] for two possible fractionation factors: In the modern Southern Ocean [79] and an average of the whole range of fractionation factors reported in the literature. The analysed OM leachate $\mathrm{Cd}$ isotopic compositions are plotted as green dashes representing the horizontal range in theoretical surface water Cd isotope compositions of which Cd was tapped by the OM. (B) Cadmium concentrations vs. Cd isotopic composition of the ambient fluid of which $\mathrm{Cd}$ was incorporated into authigenic carbonates and sulphides (light shaded symbols). Nonshaded symbols show the obtained Cd concentration and isotopic compositions analysed in the L1 (carbonate) and L4 leachates (sulphide). Error bars $=2$ SD (long-term, internal standard reproducibility).

In essence, our sequential leaching approach showed that it is possible to decipher the paleo deep and surface water $\mathrm{Cd}$ partitioning in mixed marine lithologies by analysing the authigenic phases presumably formed in variable depths of the water column. We have shown that OM in a reducing environment likely preserved the $\mathrm{Cd}$ isotope composition of phototrophic organisms taking up light $\mathrm{Cd}$ as a nutrient in the surface waters and leaving the surface water isotopically heavy. Assuming that the fractionation factor was in the order of modern ocean phytoplankton primary production, the restricted Nanhua Basin surface seawater $\varepsilon^{112 / 110} \mathrm{Cd}_{\mathrm{SSW}}=+4.5$ matches with values of the modern surface open ocean [79] and the assumed surface water composition in the early Cambrian at the same location [90]. By contrast, the leachates of early diagenetic Mn carbonates and framboidal sulphides from the basal Datangpo Fm. represent the Cd isotopic composition of Cryogenian deep water for the restricted Nanhua Basin $\left(\varepsilon^{112 / 110} \mathrm{Cd}\right.$ DW $\left.=+2.5\right)$, which was strikingly heavier than that of modern deep waters of the open ocean [54]. Applying the idea of Hohl et al. [90], we suggest that the decoupling of the DOC's Cd isotope composition and that of presumable ambient seawater was likely the result of fluctuating and progressively increasing deep water oxygenation in the early Cambrian that ultimately led to $\varepsilon^{112 / 110} \mathrm{Cd}$ values in the deep and surface waters that are in the range of modern oceans. That period of extreme ocean redox instability led to perturbations in the nutrient cycle at the Precambrian Cambrian boundary that were necessary for the evolution of animal life. 


\section{Conclusions}

The paleo-environmental reconstruction of the Nanhua Basin during the Cryogenian Period yields surface water $\mathrm{Cd}$ isotope compositions obtained in OM leachates of $\varepsilon^{112 / 110} \mathrm{Cd}_{\mathrm{SSW}}=+4.5$ that overlaps with modern surface ocean and the assumed Nanhua Basin surface water composition in the early Cambrian. Deep water, represented by authigenic carbonate and sulphide phases, were in equilibrium with bottom and pore waters and show $\varepsilon^{112 / 110} \mathrm{Cd}_{\mathrm{DW}}=+2.5$, which is heavier than that of modern, oxidized, deep ocean water masses. In the Cryogenian Nanhua Basin, the decoupling of OM and deep-water compositions was yet not achieved and the deep waters' Cd isotope composition was most likely in an equilibrium with that of deposited and partly decomposed OM. As a result, the deep water and the surface water gradient in $\varepsilon^{112 / 110} \mathrm{Cd}$ space was much smaller than in the Phanerozoic oceans and the supressed nutrient recycling may have ultimately led to the postponed evolution of animal life at the end of the Neoproterozoic.

Future research in redox-stratified Precambrian ocean sediments needs to keep these processes in mind and researchers should apply sequential leaching methods in order to obtain Cd isotope compositions of individual marine authigenic phases and the fluids that were in equilibrium with them.

Supplementary Materials: The following figures are available online http://www.mdpi.com/2076-3263/10/1/36/s1. Figure S1: SMS-19 EBSD picture of Mn carbonate beds. Figure S2: SMS-19 EBSD picture of Mn carbonates and framboidal sulphides. Figure S3: EDX maps of SMS-19 showing elemental distributions. Figure S4: Relationship between Cd concentrations obtained by standard calibration ICP-MS vs. TIMS ID. Figure S5: Relations between $\mathrm{Cd}$ isotopic compositions in leachates with fluid mobile and detrital mineral derived geochemical proxies.

Author Contributions: The research was designed on a collaborative field workshop in SW China in the year 2017 led by S.-Y.J. and S.V.H. with input by S.V. Samples were provided by S.-Y.J. and preliminary research on the samples was performed by W.W., S.V.H. processed the samples in the lab and did the trace metal analyses with the help of Q.L. at Nanjing University, while Cd isotope analyses were done in collaboration with S.J.G.G. at MPI Mainz. S.V.H. wrote the manuscript with contributions from H.-Z.W., S.-Y.J., and S.V. All authors have read and agreed to the published version of the manuscript.

Funding: This research was funded by the young international scientist fund of the National Science Foundation of China NSFC [funding Nos. 41650110480 and 41750410690].

Acknowledgments: We would like to thank the team of MPI Chemie Mainz for their help in processing and analysing Cd isotope compositions as well as Ms. Shen at the State Key Laboratory for Mineral Deposits Research, Nanjing University for the stable $\mathrm{C}$ and $\mathrm{O}$ analyses. We mourn for the tragic loss of our co-author and laboratory assistant Liu Qian whose help on trace metal concentration analyses at Nanjing University was essential to our research. We further would like to thank Susan Little and two anonymous reviewers for their valuable comments and input on an earlier version of this manuscript.

Conflicts of Interest: The authors declare no conflict of interest.

\section{References}

1. Ling, W. Neoproterozoic tectonic evolution of the northwestern Yangtze craton, South China: Implications for amalgamation and break-up of the Rodinia Supercontinent. Precambrian Res. 2003, 122, 111-140. [CrossRef]

2. Hoffman, P.F. A Neoproterozoic Snowball Earth. Science 1998, 281, 1342-1346. [CrossRef]

3. Kirschvink, J.L. Late Proterozoic low-latitude global glaciation: The snowball Earth. In The Proterozoic Biosphere: A Multidisciplinary Study; Cambridge University Press: New York, NY, USA, 1992.

4. Sahoo, S.K.; Planavsky, N.J.; Kendall, B.; Wang, X.; Shi, X. Ocean oxygenation in the wake of the Marinoan glaciation. Nature 2012, 489, 546. [CrossRef]

5. Canfield, D.E.; Poulton, S.W.; Narbonne, G.M. Late-Neoproterozoic Deep-Ocean Oxygenation and the Rise of Animal Life. Science 2007, 315, 92-95. [CrossRef]

6. Narbonne, G.M. Ocean Chemistry and Early Animals. Science 2010, 328, 53-54. [CrossRef]

7. Cai, Y.; Xiao, S.; Li, G.; Hua, H. Diverse biomineralizing animals in the terminal Ediacaran Period herald the Cambrian explosion. Geology 2019, 47, 380-384. [CrossRef]

8. Xiao, S.; Zhang, Y.; Knoll, A.H. Three-dimensional preservation of algae and animal embryos in a Neoproterozoic phosphorite. Nature 1998, 391, 593. [CrossRef] 
9. Lenton, T.M.; Boyle, R.A.; Poulton, S.W.; Shields-Zhou, G.A.; Butterfield, N.J. Co-evolution of eukaryotes and ocean oxygenation in the Neoproterozoic era. Nat. Geosci. 2014, 7, 257-265. [CrossRef]

10. Li, C.; Love, G.D.; Lyons, T.W.; Scott, C.T.; Feng, L.; Huang, J.; Chang, H.; Zhang, Q.; Chu, X. Evidence for a redox stratified Cryogenian marine basin, Datangpo Formation, South China. Earth Planet. Sci. Lett. 2012, 331, 246-256. [CrossRef]

11. Zhang, F.; Zhu, X.; Yan, B.; Kendall, B.; Peng, X.; Li, J.; Algeo, T.J.; Romaniello, S. Oxygenation of a Cryogenian ocean (Nanhua Basin, South China) revealed by pyrite Fe isotope compositions. Earth Planet. Sci. Lett. 2015, 429, 11-19. [CrossRef]

12. Li, C.; Love, G.D.; Lyons, T.W.; Fike, D.A.; Sessions, A.L.; Chu, X. A Stratified Redox Model for the Ediacaran Ocean. Science 2010, 328, 80-83. [CrossRef] [PubMed]

13. Wood, R.A.; Poulton, S.W.; Prave, A.R.; Hoffmann, K.H.; Clarkson, M.O.; Guilbaud, R.; Lyne, J.W.; Tostevin, R.; Bowyer, F.; Penny, A.M.; et al. Dynamic redox conditions control late Ediacaran metazoan ecosystems in the Nama Group, Namibia. Precambrian Res. 2015, 261, 252-271. [CrossRef]

14. Wood, R.; Erwin, D.H. Innovation not recovery: Dynamic redox promotes metazoan radiations. Biol. Rev. 2017, 93, 863-873. [CrossRef] [PubMed]

15. Love, G.D.; Grosjean, E.; Stalvies, C.; Fike, D.A.; Grotzinger, J.P.; Bradley, A.S.; Kelly, A.E.; Bhatia, M.; Meredith, W.; Snape, C.E.; et al. Fossil steroids record the appearance of Demospongiae during the Cryogenian period. Nature 2009, 457, 718. [CrossRef] [PubMed]

16. Knoll, A.H. Biogeochemistry: Food for early animal evolution. Nature 2017, 548, 528-530. [CrossRef] [PubMed]

17. Brocks, J.J.; Jarrett, A.J.M.; Sirantoine, E.; Hallmann, C.; Hoshino, Y.; Liyanage, T. The rise of algae in Cryogenian oceans and the emergence of animals. Nature 2017, 548, 578-581. [CrossRef]

18. Zhou, J.-C.; Wang, X.-L.; Qiu, J.-S. Geochronology of Neoproterozoic mafic rocks and sandstones from northeastern Guizhou, South China: Coeval arc magmatism and sedimentation. Precambrian Res. 2009, 170, 27-42. [CrossRef]

19. Zhang, S.; Jiang, G.; Han, Y. The age of the Nantuo Formation and Nantuo glaciation in South China. Terra Nova 2008, 20, 289-294. [CrossRef]

20. Condon, D. U-Pb Ages from the Neoproterozoic Doushantuo Formation, China. Science 2005, 308, 95-98. [CrossRef]

21. Cheng, M.; Li, C.; Chen, X.; Zhou, L.; Algeo, T.J.; Ling, H.-F.; Feng, L.-J.; Jin, C.-S. Delayed Neoproterozoic oceanic oxygenation_Evidence from Mo isotopes of the Cryogenian Datangpo Formation. Precambrian Res. 2018, 319, 187-197. [CrossRef]

22. Ye, Y.; Wang, H.; Zhai, L.; Wang, X.; Wu, C.; Zhang, S. Contrasting Mo-U enrichments of the basal Datangpo Formation in South China: Implications for the Cryogenian interglacial ocean redox. Precambrian Res. 2018, 315, 66-74. [CrossRef]

23. Ma, Z.; Liu, X.; Yu, W.; Du, Y.; Du, Q. Redox conditions and manganese metallogenesis in the Cryogenian Nanhua Basin: Insight from the basal Datangpo Formation of South China. Palaeogeogr. Palaeoclimatol. Palaeoecol. 2019, 529, 39-52. [CrossRef]

24. Xiao, J.; He, J.; Yang, H.; Wu, C. Comparison between Datangpo-type manganese ores and modern marine ferromanganese oxyhydroxide precipitates based on rare earth elements. Ore Geol. Rev. 2017, 89, 1-46. [CrossRef]

25. Peng, X.; Zhu, X.-K.; Shi, F.; Yan, B.; Zhang, F.; Zhao, N.; Peng, P.; Li, J.; Wang, D.; Shields, G.A. A deep marine organic carbon reservoir in the non-glacial Cryogenian ocean (Nanhua Basin, South China) revealed by organic carbon isotopes. Precambrian Res. 2019, 321, 212-220. [CrossRef]

26. Wei, W.; Wang, D.; Li, D.; Ling, H.; Chen, X.; Wei, G.; Zhang, F.; Zhu, X.; Yan, B. The marine redox change and nitrogen cycle in the Early Cryogenian interglacial time: Evidence from nitrogen isotopes and Mo contents of the basal Datangpo Formation, northeastern Guizhou, South China. J. Earth Sci. 2016, 27, 233-241. [CrossRef]

27. Anbar, A.D. Elements and Evolution. Science 2008, 322, 1481-1483. [CrossRef]

28. Sunda, W.G. Trace Element Nutrients; Elsevier: Amsterdam, The Netherlands, 2001; pp. 75-86.

29. Whitfield, M. The mean oceanic residence time (MORT) concept-A rationalisation. Mar. Chem. 1979, 8, 101-123. [CrossRef] 
30. Viehmann, S.; Hohl, S.V.; Kraemer, D.; Bau, M.; Walde, D.H.G.; Galer, S.J.G.; Jiang, S.-Y.; Meister, P. Metal cycling in Mesoproterozoic microbial habitats: Insights from trace elements and stable Cd isotopes in stromatolites. Gondwana Res. 2019, 67, 1-14. [CrossRef]

31. Viehmann, S.; Bau, M.; Hoffmann, J.E.; Münker, C. Decoupled Hf and Nd isotopes in suspended particles and in the dissolved load of Late Archean seawater. Chem. Geol. 2018, 483, 111-118. [CrossRef]

32. Schier, K.; Bau, M.; Münker, C.; Beukes, N.; Viehmann, S. Trace element and Nd isotope composition of shallow seawater prior to the Great Oxidation Event: Evidence from stromatolitic bioherms in the Paleoproterozoic Rooinekke and Nelani Formations, South Africa. Precambrian Res. 2018, 315, 1-43. [CrossRef]

33. Viehmann, S.; Hoffmann, J.E.; Münker, C.; Bau, M. Decoupled Hf-Nd isotopes in Neoarchean seawater reveal weathering of emerged continents. Geology 2014, 42, 115-118. [CrossRef]

34. Viehmann, S.; Bau, M.; Hoffmann, J.E.; Münker, C. Geochemistry of the Krivoy Rog Banded Iron Formation, Ukraine, and the impact of peak episodes of increased global magmatic activity on the trace element composition of Precambrian seawater. Precambrian Res. 2015, 270, 165-180. [CrossRef]

35. Canfield, D.E. A new model for Proterozoic ocean chemistry. Nature 1998, 396, 450. [CrossRef]

36. Morse, J.W.; Mackenzie, F.T. Geochemistry of Sedimentary Carbonates; Elsevier: Amsterdam, The Netherlands, 1990.

37. Hohl, S.V.; Becker, H.; Jiang, S.-Y.; Ling, H.-F.; Guo, Q.; Struck, U. Geochemistry of Ediacaran cap dolostones across the Yangtze Platform, South China: Implications for diagenetic modification and seawater chemistry in the aftermath of the Marinoan glaciation. J. Geol. Soc. 2017, 174, 893-912. [CrossRef]

38. Tostevin, R.; Shields, G.A.; Tarbuck, G.M.; He, T.; Clarkson, M.O.; Wood, R.A. Effective use of cerium anomalies as a redox proxy in carbonate-dominated marine settings. Chem. Geol. 2016, 438, 146-162. [CrossRef]

39. Wu, H.-P.; Jiang, S.-Y.; Palmer, M.R.; Wei, H.-Z.; Yang, J.-H. Positive cerium anomaly in the Doushantuo cap carbonates from the Yangtze platform, South China: Implications for intermediate water column manganous conditions in the aftermath of the Marinoan glaciation. Precambrian Res. 2018, 320, 93-110. [CrossRef]

40. Thomson, J.; Higgs, N.C.; Jarvis, I.; Hydes, D.J. The behaviour of manganese in Atlantic carbonate sediments. Geochim. Et Cosmochim. Acta 1986, 50, 1807-1818. [CrossRef]

41. Tribovillard, N.; Algeo, T.J.; Lyons, T.; Riboulleau, A. Trace metals as paleoredox and paleoproductivity proxies: An update. Chem. Geol. 2006, 232, 12-32. [CrossRef]

42. Horner, T.J.; Kinsley, C.W.; Nielsen, S.G. Barium-isotopic fractionation in seawater mediated by barite cycling and oceanic circulation. Earth Planet. Sci. Lett. 2015, 430, 511-522. [CrossRef]

43. Morford, J.L.; Emerson, S. The geochemistry of redox sensitive trace metals in sediments. Geochim. Et Cosmochim. Acta 1999, 63, 1735-1750. [CrossRef]

44. John, S.G.; Rouxel, O.J.; Craddock, P.R.; Engwall, A.M.; Boyle, E.A. Zinc stable isotopes in seafloor hydrothermal vent fluids and chimneys. Earth Planet. Sci. Lett. 2008, 269, 17-28. [CrossRef]

45. Guinoiseau, D.; Galer, S.J.G.; Abouchami, W. Effect of cadmium sulphide precipitation on the partitioning of Cd isotopes: Implications for the oceanic Cd cycle. Earth Planet. Sci. Lett. 2018, 498, 300-308. [CrossRef]

46. Janssen, D.J.; Conway, T.M.; John, S.G.; Christian, J.R.; Kramer, D.I.; Pedersen, T.F.; Cullen, J.T. Undocumented water column sink for cadmium in open ocean oxygen-deficient zones. Proc. Natl. Acad. Sci. USA 2014, 111, 6888-6893. [CrossRef] [PubMed]

47. Large, R.R.; Halpin, J.A.; Lounejeva, E.; Danyushevsky, L.V.; Maslennikov, V.V.; Gregory, D.; Sack, P.J.; Haines, P.W.; Long, J.A.; Makoundi, C.; et al. Cycles of nutrient trace elements in the Phanerozoic ocean. Gondwana Res. 2015, 28, 1282-1293. [CrossRef]

48. Tribovillard, N.; Algeo, T.J.; Baudin, F.; Riboulleau, A. Analysis of marine environmental conditions based onmolybdenum-uranium covariation-Applications to Mesozoic paleoceanography. Chem. Geol. 2012, 324, 46-58. [CrossRef]

49. Algeo, T.J.; Tribovillard, N. Environmental analysis of paleoceanographic systems based on molybdenum-uranium covariation. Chem. Geol. 2009, 268, 211-225. [CrossRef]

50. Little, S.H.; Vance, D.; Lyons, T.W.; McManus, J. Controls on trace metal authigenic enrichment in reducing sediments: Insights from modern oxygen-deficient settings. Am. J. Sci. 2015, 315, 77-119. [CrossRef]

51. Hohl, S.V.; Becker, H.; Herzlieb, S.; Guo, Q. Multiproxy constraints on alteration and primary compositions of Ediacaran deep-water carbonate rocks, Yangtze Platform, South China. Geochim. Et Cosmochim. Acta 2015, 163, 262-278. [CrossRef] 
52. Smrzka, D.; Zwicker, J.; Bach, W.; Feng, D.; Himmler, T.; Chen, D.; Peckmann, J. The behavior of trace elements in seawater, sedimentary pore water, and their incorporation into carbonate minerals: A review. Facies 2019, 65, 1-47. [CrossRef]

53. Abouchami, W.; Galer, S.J.G.; de Baar, H.J.W.; Alderkamp, A.C.; Middag, R.; Laan, P.; Feldmann, H.; Andreae, M.O. Modulation of the Southern Ocean cadmium isotope signature by ocean circulation and primary productivity. Earth Planet. Sci. Lett. 2011, 305, 83-91. [CrossRef]

54. Ripperger, S.; Rehkämper, M.; Porcelli, D.; Halliday, A.N. Cadmium isotope fractionation in seawater-A signature of biological activity. Earth Planet. Sci. Lett. 2007, 261, 670-684. [CrossRef]

55. Lacan, F.; Francois, R.; Ji, Y.; Sherrell, R.M. Cadmium isotopic composition in the ocean. Geochim. Et Cosmochim. Acta 2006, 70, 5104-5118. [CrossRef]

56. Schmitt, A.-D.; Galer, S.J.G.; Abouchami, W. Mass-dependent cadmium isotopic variations in nature with emphasis on the marine environment. Earth Planet. Sci. Lett. 2009, 277, 262-272. [CrossRef]

57. Hohl, S.V.; Galer, S.J.G.; Gamper, A.; BECKER, H. Cadmium isotope variations in Neoproterozoic carbonates-A tracer of biologic production? Geochem. Persp. Lett. 2016, 3, 32-44. [CrossRef]

58. John, S.G.; Kunzmann, M.; Townsend, E.J.; Rosenberg, A.D. Zinc and cadmium stable isotopes in the geological record: A case study from the post-snowball Earth Nuccaleena cap dolostone. Palaeogeogr. Palaeoclimatol. Palaeoecol. 2017, 466, 202-208. [CrossRef]

59. Price, N.M.; Morel, F.M.M. Cadmium and cobalt substitution for zinc in a marine diatom. Nature 1990, 344, 658-660. [CrossRef]

60. Xu, Y.; Feng, L.; Jeffrey, P.D.; Shi, Y.; Morel, F.M.M. Structure and metal exchange in the cadmium carbonic anhydrase of marine diatoms. Nature 2008, 452, 56-61. [CrossRef]

61. Georgiev, S.V.; Horner, T.J.; Stein, H.J.; Hannah, J.L. Cadmium-isotopic evidence for increasing primary productivity during the Late Permian anoxic event. Earth Planet. Sci. Lett. 2015, 410, 84-96. [CrossRef]

62. Schmitt, A.-D.; Galer, S.J.G.; Abouchami, W. High-precision cadmium stable isotope measurements by double spike thermal ionisation mass spectrometry. J. Anal. At. Spectrom. 2009, 24, 1079-1088. [CrossRef]

63. Zhang, Y.; Wen, H.; Zhu, C.; Fan, H.; Cloquet, C. Cadmium isotopic evidence for the evolution of marine primary productivity and the biological extinction event during the Permian-Triassic crisis from the Meishan section, South China. Chem. Geol. 2018, 481, 110-118. [CrossRef]

64. Horner, T.J.; Rickaby, R.E.M.; Henderson, G.M. Isotopic fractionation of cadmium into calcite. Earth Planet. Sci. Lett. 2011, 312, 243-253. [CrossRef]

65. Horner, T.J. Cadmium Isotope Fractionation in Seawater. Ph.D. Thesis, The University of Oxford, Oxford, UK, 2012.

66. Lambelet, M.; Rehkämper, M.; van de Flierdt, T.; Xue, Z.; Kreissig, K.; Coles, B.; Porcelli, D.; Andersson, P. Isotopic analysis of $\mathrm{Cd}$ in the mixing zone of Siberian rivers with the Arctic Ocean-New constraints on marine Cd cycling and the isotope composition of riverine Cd. Earth Planet. Sci. Lett. 2013, 361, 64-73. [CrossRef]

67. Wei, G.-Y.; Planavsky, N.J.; Tarhan, L.G.; Chen, X.; Wei, W.; Li, D.; Ling, H.-F. Marine redox fluctuation as a potential trigger for the Cambrian explosion. Geology 2018, 46, 1-5. [CrossRef]

68. Henrique-Pinto, R.; Barnes, S.-J.; Savard, D.D.; Mehdi, S. Quantification of Metals and Semi-Metals in Carbon-Rich Rocks: A New Sequential Protocol Including Extraction from Humic Substances. Geostand. Geoanalytical Res. 2015, 41, 41-62. [CrossRef]

69. Zhang, Q.R.; Chu, X.L.; Bahlburg, H.; Feng, L.J.; Dobrzinski, N.; Zhang, T.G. Stratigraphic architecture of the neoproterozoic glacial rocks in the "Xiang-Qian-Gui" region of the central Yangtze block, South China. Prog. Nat. Sci. 2003, 13, 783-787. [CrossRef]

70. Jiang, G.; Shi, X.; Zhang, S.; Wang, Y.; Xiao, S. Stratigraphy and paleogeography of the Ediacaran Doushantuo Formation (ca. 635-551Ma) in South China. Gondwana Res. 2011, 19, 831-849. [CrossRef]

71. Boguta, P.; Sokołowska, Z. Interactions of Zn(II) Ions with Humic Acids Isolated from Various Type of Soils. Effect of pH, Zn Concentrations and Humic Acids Chemical Properties. PLoS ONE 2016, 11, e015362626. [CrossRef]

72. Govindaraju, K. 1994 compilation of working values and sample description for 383 Geostandards. Geostand. Newsl. 1994, 18, 1-158. [CrossRef]

73. Rosman, K.J.R.; De Laeter, J.R.; Gorton, M.P. Cadmium isotope fractionation in fractions of two H3 chondrites. Earth Planet. Sci. Lett. 1980, 48, 166-170. [CrossRef] 
74. Abouchami, W.; Galer, S.J.G.; Horner, T.J.; Rehkämper, M.; Wombacher, F.; Xue, Z.; Lambelet, M.; Gault-Ringold, M.; Stirling, C.H.; Schönbächler, M.; et al. A Common Reference Material for Cadmium Isotope Studies-NIST SRM 3108. Geostand. Geoanal. Res. 2012, 37, 5-17. [CrossRef]

75. McCrea, J.M. On the Isotopic Chemistry of Carbonates and a Paleotemperature Scale. J. Chem. Phys. 1950, 18, 849-857. [CrossRef]

76. Coplen, T.B. Guidelines and recommended terms for expression of stable-isotope-ratio and gas-ratio measurement results. Rapid Commun. Mass Spectrom. 2011, 25, 2538-2560. [CrossRef] [PubMed]

77. Taylor, S.R.; McLennan, S.M. The Continental Crust; Wiley-Blackwell: Hoboken, NJ, USA, 1991.

78. McLennan, S.M. Relationships between the trace element composition of sedimentary rocks and upper continental crust. Geochem. Geophys. Geosystems 2001, 2. [CrossRef]

79. Abouchami, W.; Galer, S.J.G.; de Baar, H.J.W.; Middag, R.; Vance, D.; Zhao, Y.; Klunder, M.; Mezger, K.; Feldmann, H.; Andreae, M.O. Biogeochemical cycling of cadmium isotopes in the Southern Ocean along the Zero Meridian. Geochim. Et Cosmochim. Acta 2014, 127, 348-367. [CrossRef]

80. Sieber, M.; Conway, T.M.; de Souza, G.F.; Hassler, C.S.; Ellwood, M.J.; Vance, D. High-resolution Cd isotope systematics in multiple zones of the Southern Ocean from the Antarctic Circumnavigation Expedition. Earth Planet. Sci. Lett. 2019, 527, 115799. [CrossRef]

81. Conway, T.M.; John, S.G. Biogeochemical cycling of cadmium isotopes along a high-resolution section through the North Atlantic Ocean. Geochim. Et Cosmochim. Acta 2015, 148, 269-283. [CrossRef]

82. Horner, T.J.; Schönbächler, M.; Rehkämper, M.; Nielsen, S.G.; Williams, H.; Halliday, A.N.; Xue, Z.; Hein, J.R. Ferromanganese crusts as archives of deep water Cd isotope compositions. Geochem. Geophys. Geosyst. 2010, 11, 4. [CrossRef]

83. Cronan, D.S. Underwater Minerals; Academic Press: Cambridge, MA, USA, 1980.

84. Comans, R. Adsorption, desorption and isotopic exchange of cadmium on illite: Evidence for complete reversibility. Water Res. 1987, 21, 1573-1576. [CrossRef]

85. Xie, R.C.; Rehkämper, M.; Grasse, P.; van de Flierdt, T.; Frank, M.; Xue, Z. Isotopic evidence for complex biogeochemical cycling of Cd in the eastern tropical South Pacific. Earth Planet. Sci. Lett. 2019, 512, 134-146. [CrossRef]

86. Xie, R.C.; Galer, S.J.G.; Abouchami, W.; Rijkenberg, M.J.A.; de Baar, H.J.W.; De Jong, J.; Andreae, M.O. Non-Rayleigh control of upper-ocean Cd isotope fractionation in the western South Atlantic. Earth Planet. Sci. Lett. 2017, 471, 94-103. [CrossRef]

87. George, E. Marine Biogeochemical Cycling of Cadmium and Its Isotopes: Studies of the South Pacific Ocean, Mediterranean Sea and Black Sea. Ph.D. Thesis, University of Otago, Dunedin, New Zealand, 2017. Available online: http://hdl.handle.net/10523/7552 (accessed on 8 January 2020).

88. George, E.; Stirling, C.H.; Gault-Ringold, M.; Ellwood, M.J.; Middag, R. Marine biogeochemical cycling of cadmium and cadmium isotopes in the extreme nutrient-depleted subtropical gyre of the South West Pacific Ocean. Earth Planet. Sci. Lett. 2019, 514, 84-95. [CrossRef]

89. Boyle, E.A. Cadmium: Chemical tracer of deepwater paleoceanography. Paleoceanography 1988, 3, 471-489. [CrossRef]

90. Hohl, S.V.; Jiang, S.-Y.; Wei, H.-Z.; Pi, D.-H.; Liu, Q.; Viehmann, S.; Galer, S.J.G. Cd isotopes trace periodic (bio)geochemical metal cycling at the verge of the Cambrian animal evolution. Geochim. Et Cosmochim. Acta 2019, 263, 1-20. [CrossRef]

91. Xie, R.C.; Galer, S.J.G.; Abouchami, W.; Frank, M. Limited impact of eolian and riverine sources on the biogeochemical cycling of Cd in the tropical Atlantic. Chem. Geol. 2019, 511, 371-379. [CrossRef]

(C) 2020 by the authors. Licensee MDPI, Basel, Switzerland. This article is an open access article distributed under the terms and conditions of the Creative Commons Attribution (CC BY) license (http://creativecommons.org/licenses/by/4.0/). 